\title{
Aging of Preleukemic Thymocytes Drives CpG Island Hypermethylation in T-cell Acute' Lymphoblastic Leukemia
}

Juliette Roels $s^{1,2}$, Morgan Thénoz $z^{1,2}$, Bronisława Szarzyńska ${ }^{3}$, Mattias Landfors ${ }^{4}$, Stien De Coninck ${ }^{1,2}$, Lisa Demoen ${ }^{1,2}$, Lien Provez ${ }^{1,2}$, Anna Kuchmiy ${ }^{2,5}$, Steven Strubbe ${ }^{5}$, Lindy Reunes ${ }^{1,2}$, Tim Pieters ${ }^{1,2}$, Filip Matthijssens ${ }^{1,2}$, Wouter Van Loocke ${ }^{1,2}$, Büşra Erarslan-Uysal ${ }^{6,7}$, Paulina Richter-Pechańska ${ }^{6,7}$, Ken Declerck ${ }^{8}$, Tim Lammens ${ }^{2,9}$, Barbara De Moerloose ${ }^{2,9}$, Dieter Deforce ${ }^{10}$. Filip Van Nieuwerburgh ${ }^{10}$, Laurence C. Cheung ${ }^{11,12}$, Rishi S. Kotecha ${ }^{11,12}$, Marc R. Mansour ${ }^{13}$, Bart Ghesquière ${ }^{14}$, Guy Van Camp ${ }^{15}$, Wim Vanden Berghe ${ }^{8}$, Jerzy R. Kowalczyk ${ }^{16}$, Tomasz Szczepański ${ }^{17}$, Utpal P. Davé ${ }^{18}$, Andreas E. Kulozik ${ }^{6,7}$, Steven Goossens ${ }^{1,2,5}$, David J. Curtis ${ }^{19}$, Tom Taghon ${ }^{5}$, Małgorzata Dawidowskä ${ }^{3}$, Sofie Degerman ${ }^{4,20}$; and Pieter Van Vlierberghe ${ }^{1,2}$
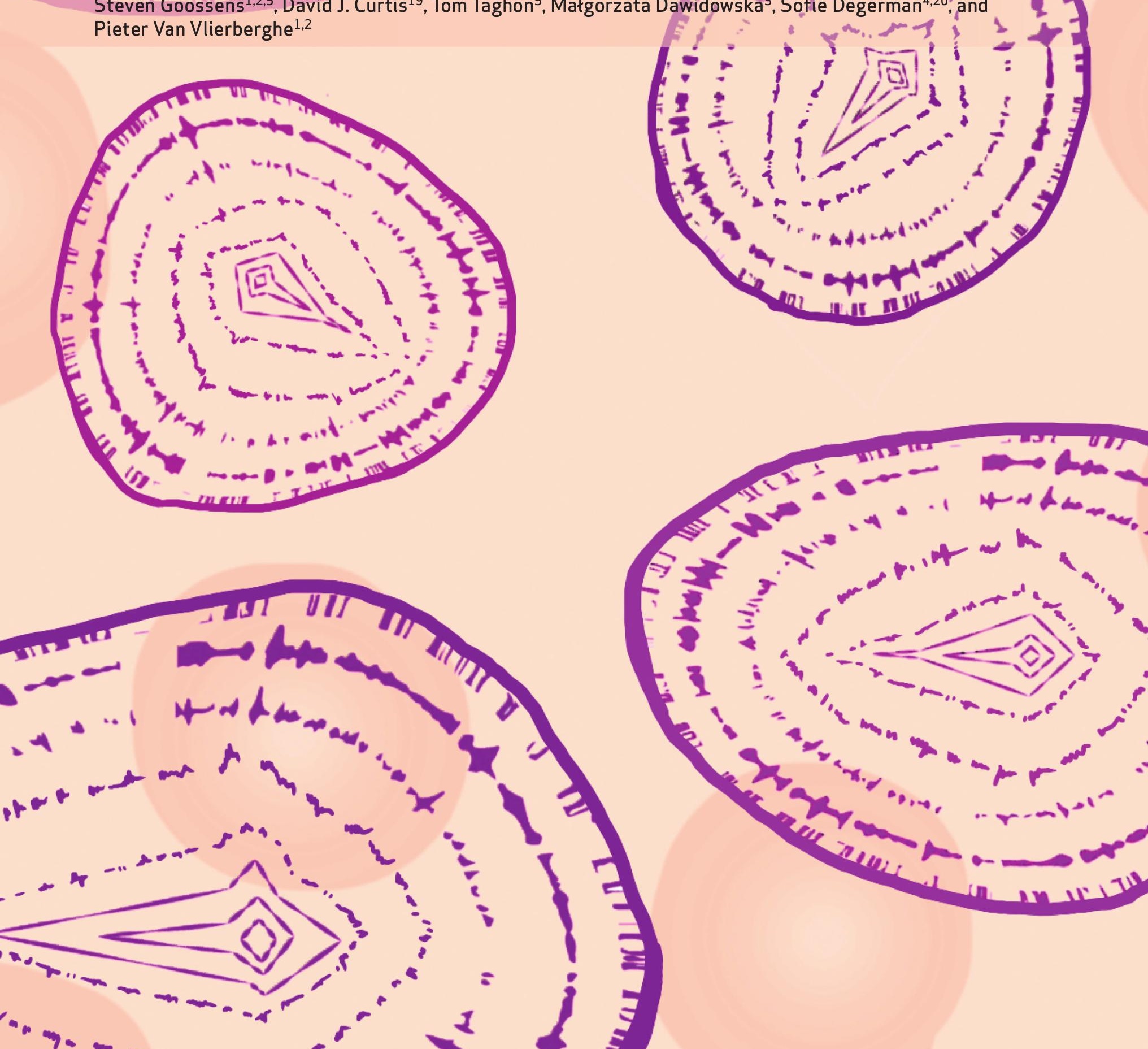
ABSTRACT Cancer cells display DNA hypermethylation at specific CpG islands in comparison with their normal healthy counterparts, but the mechanism that drives this socalled $\mathrm{CpG}$ island methylator phenotype (CIMP) remains poorly understood. Here, we show that $\mathrm{CpG}$ island methylation in human T-cell acute lymphoblastic leukemia (T-ALL) mainly occurs at promoters of Polycomb Repressor Complex 2 (PRC2) target genes that are not expressed in normal or malignant T cells and that display a reciprocal association with H3K27me3 binding. In addition, we reveal that this aberrant methylation profile reflects the epigenetic history of T-ALL and is established already in preleukemic, self-renewing thymocytes that precede T-ALL development. Finally, we unexpectedly uncover that this age-related $\mathrm{CpG}$ island hypermethylation signature in T-ALL is completely resistant to the FDA-approved hypomethylating agent decitabine. Altogether, we provide conceptual evidence for the involvement of a preleukemic phase characterized by self-renewing thymocytes in the pathogenesis of human T-ALL.

SIGNIFICANCE: We developed a DNA methylation signature that reveals the epigenetic history of thymocytes during $\mathrm{T}$-cell transformation. This human signature was recapitulated by murine self-renewing preleukemic thymocytes that build an age-related $\mathrm{CpG}$ island hypermethylation phenotype, providing conceptual evidence for the involvement of a preleukemic thymic phase in human T-cell leukemia.

\section{INTRODUCTION}

During the last decade, aberrant DNA methylation has been identified as a hallmark of human cancer and several studies have highlighted the promising potential of DNA methylation as a clinically or diagnostically relevant biomarker (1). In comparison with their putative normal healthy counterparts, cancer cells generally display DNA hypermethylation at specific CPG islands, but the actual mechanism that drive this so-called $\mathrm{CpG}$ island methylator phenotype (CIMP) remains poorly understood (2). Despite the fact that DNA hypomethylating agents are actively used in the clinic for the treatment of some hematologic malignancies, their putative effects on leukemia-specific DNA methylation signatures and gene expression remain unclear $(1,2)$.

T-cell acute lymphoblastic leukemia (T-ALL) is an aggressive hematologic cancer for which prognostically relevant CIMP subtypes have also been described (3). Indeed, based on the methylation status of about 1,000 promoter-associated
CpG sites, $\mathrm{CIMP}^{+} \mathrm{T}$-ALLs were associated with a better eventfree and overall survival as compared to $\mathrm{CIMP}^{-}$leukemias (3). Notably, these findings were recently confirmed in independent pediatric (4) and adult (5) T-ALL cohorts, further reinforcing the idea that aberrant DNA methylation might act as a clinically relevant biomarker in human T-ALL. However, the mechanism driving the CIMP in T-cell leukemia has remained elusive so far.

In this study, we performed a comprehensive DNA methylome analysis of normal and malignant $\mathrm{T}$ cells in both human and murine settings in an attempt to increase our understanding on the origin of aberrant DNA methylation in T-ALL.

\section{RESULTS}

\section{A CpG Island and Open Sea DNA Methylation Signature in Human T-ALL}

Previous studies have shown that molecular genetic subtypes of human T-ALL are associated with an arrest at specific

\footnotetext{
1Department of Biomolecular Medicine, Ghent University, Ghent, Belgium. ${ }^{2}$ Cancer Research Institute Ghent (CRIG), Ghent, Belgium. Institute of Human Genetics, Polish Academy of Sciences, Poznań, Poland. ${ }^{4}$ Department of Medical Biosciences, Umeå University, Umeå, Sweden. ${ }^{5}$ Department of Diagnostic Sciences, Ghent University, Ghent, Belgium. ${ }^{6}$ Department of Pediatric Oncology, Hematology, and Immunology, University of Heidelberg, and Hopp Children's Cancer Center at NCT Heidelberg, Heidelberg, Germany. ${ }^{7}$ Molecular Medicine Partnership Unit (MMPU), European Molecular Biology Laboratory (EMBL), University of Heidelberg, Heidelberg, Germany. ${ }^{8}$ Laboratory of Protein Chemistry, Proteomics and Epigenetic Signaling (PPES) and Integrated Personalized and Precision Oncology Network (IPPON), Department of Biomedical Sciences, University of Antwerp, Antwerp, Belgium. ${ }^{9}$ Department of Pediatric Hematology-Oncology and Stem Cell Transplantation, Ghent University Hospital, Ghent, Belgium. ${ }^{10}$ Laboratory of Pharmaceutical Biotechnology, Ghent University, Ghent, Belgium. ${ }^{11}$ Telethon Kids Cancer Centre, Telethon Kids Institute, University of Western Australia, Perth, Western Australia. ${ }^{12}$ School of Pharmacy and Biomedical Sciences, Curtin University, Perth, Western Australia. ${ }^{13}$ Department of Haematology, University College London Cancer Institute, London, England. ${ }^{14}$ Metabolomics Expertise Center, VIB Center for Cancer Biology, Leuven,
}

Belgium. ${ }^{15}$ Center of Medical Genetics, University of Antwerp, Antwerp, Belgium. ${ }^{16}$ Department of Pediatric Hematology, Oncology and Transplantology, Medical University of Lublin, Lublin, Poland. ${ }^{17}$ Department of Pediatric Hematology and Oncology, Zabrze, Medical University of Silesia, Katowice, Poland. ${ }^{18}$ Roudebush Veterans Affairs Medical Center and Indiana University School of Medicine, Indianapolis, Indiana. ${ }^{19}$ Australian Centre for Blood Diseases (ACBD), Monash University, Melbourne, Australia. ${ }^{20}$ Department of Clinical Microbiology, Umeå University, Umeå, Sweden.

Note: Supplementary data for this article are available at Blood Cancer Discovery Online (https://bloodcancerdiscov.aacrjournals.org/).

J. Roels and M. Thénoz contributed equally to this article.

Corresponding Author: Pieter Van Vlierberghe, Ghent University, Corneel Heymanslaan 10, Ghent 9000, Belgium. Phone: 329-332-1043; E-mail: pieter.vanvlierberghe@ugent.be

Blood Cancer Discov 2020;1:274-89

doi: 10.1158/2643-3230.BCD-20-0059

(C) 2020 American Association for Cancer Research. 
stages of normal human T-cell differentiation $(6,7)$. Here, we performed DNA methylation profiling using the 850k EPIC array platform on 109 primary T-ALLs (ref. 8; Supplementary Table S1) and 10 stages of sorted human thymocytes (9, $10)$, reflecting the normal counterparts of this disease (refs. 6, 7; Supplementary Table S2). Using unsupervised clustering of the 5,000 most variably methylated CpGs, we built a methylation-based signature that we termed COSMe (CpG island and Open Sea Methylation), referring to CpGs located inside CpG islands ( $n=2,713)$, CpGs located outside CpG islands $(n=1,245)$, and CpGs that flank CpG islands (CpG shores and shelves, $n=1,042$; Supplementary Table S3). This COSMe signature divides T-ALLs in two methylation-based categories, that is, COSMe type I and type II (Fig. 1A; Supplementary Table S3), based on three main clusters of CPGs (cluster A, B, and C). The subdivision of T-ALLs in COSMe-I and COSMe-II is dominated by sites located in cluster A, which are mainly located at $\mathrm{CPG}$ islands and largely correspond to the CIMP classification that has previously been established in T-ALL (ref. 3; Fig. 1A; Supplementary Table $\mathrm{S} 3)$. In line with previous reports on the clinical relevance of CIMP status in both pediatric $(3,4)$ and adult (5) T-ALL, we also observed a significantly higher cumulative incidence of relapse in the $\mathrm{CIMP}^{-} \mathrm{T}$-cell leukemias from our cohort treated according to the ALL IC-BFM 2002/2009 protocol $(P=0.04$; Supplementary Fig. S1; Supplementary Tables S4 and S5).

At the genetic level, the COSMe subtypes were differentially enriched for genetic defects previously associated with T-ALL biology and CIMP status (refs. 7, 8, 11; Fig. 1A; Supplementary Fig. S2). COSMe-I T-ALLs were significantly enriched for TAL1 rearrangements, whereas COSMe-II T-ALLs mainly consisted of leukemias with aberrant activation of TLX1, TLX3, NKX2.1, or HOXA (Fig. 1A; Supplementary Fig. S2; Supplementary Table S6; ref. 8). In addition, other genetic defects, which have previously been associated with the TAL1 gene expression cluster (7), were also more prevalent in COSMe-I T-ALLs, including $6 \mathrm{q}$ deletions $(P<0.01)$ and PTEN deletions $(P=0.071$; Fig. $1 \mathrm{~A}$; Supplementary Fig. S2; Supplementary Table S6). In contrast, COSMe-II T-ALLs showed enrichment for genetic aberrations previously associated with double-negative or early-cortical T-ALLs $(12,13)$, including $5 q$ deletions and loss-of-function alterations targeting WT1, CTCF, and the Polycomb Repressor Complex 2 (PRC2) members EZH2, SUZ12, or EED (Fig. 1A; Supplementary Fig. S2; Supplementary Table S6).

COSMe-I T-ALLs showed characteristic low-level methylation compared with COSMe-II T-ALLs in the CpG island- dominated cluster A (Fig. 1A). Nevertheless, COSMe-I T-ALLs already displayed increased DNA methylation of these cluster A CpGs in comparison with normal developing $\mathrm{T}$ cells, which uniformly lack any methylation at these sites (Fig. 1B).

Besides cluster A, the COSMe signature also included two clusters that mainly consisted of Open Sea CpG sites (clusters B and C; Supplementary Table S3; Fig. 1A). Remarkably, cluster B methylation gradually increased during T-cell development (Fig. 1C). Cluster B CpG sites displayed hypermethylation in almost all COSMe-I T-ALLs but in only a subset of COSMe-II leukemias (Fig. 1A and C).

To further study this, we performed clustering of COSMe-II leukemias solely based on cluster B methylation levels and identified COSMe-II cluster $\mathrm{B}^{+}$and COSMe-II cluster $\mathrm{B}^{-}$T-ALLs (Supplementary Fig. S3A). Of note, COSMe-II cluster B- leukemias showed a trend for enrichment of genetic defects previously associated with early immature T-ALL and early T-cell precursor acute lymphoblastic leukemia (ETP-ALL; refs. 12, 13), including $5 \mathrm{q}$ deletions, loss-of-function alterations targeting WT1, CTCF, PRC2 (EZH2, SUZ12, or EED), as well as leukemias displaying aberrant activation of HOXA genes (Supplementary Fig. S3A and $\mathrm{S} 3 \mathrm{~B}$ ). In contrast, there was a trend for higher prevalence of $6 \mathrm{q}$ deletions, loss-of-function alterations targeting PTEN, as well as T-ALLs showing aberrant expression of NKX2.1 in COSMe-II cluster $\mathrm{B}^{+}$leukemias (Supplementary Fig. S3A and S3B).

Finally, and in contrast to clusters A and B, cluster C CPG methylation was more heterogeneous in normal $\mathrm{T}$ cells and both COSMe T-ALL subtypes (Fig. 1A).

To further validate these findings, we subsequently profiled an independent cohort of 14 patients with T-ALL and two thymocyte subsets $\left(\mathrm{CD} 34^{+}\right.$and $\left.\mathrm{CD} 4^{+} \mathrm{CD} 8^{+}\right)$by EPIC sequencing (EPIC-seq), an alternative, sequencing-based DNA methylation profiling method (Supplementary Fig. S4A-S4C). Clustering using the COSMe signature (Supplementary Table S3) confirmed the presence of COSMe-I and COSMe-II T-ALLs, with TAL1-rearranged T-ALLs being exclusively present in the COSMe-I subtype (Supplementary Fig. S4A). Furthermore, in this series, COSMe-II T-ALLs consisted of immature ETPALL as well as $T L X 1^{+}, T L X 3^{+}, N K X 2.1^{+}$, or $\mathrm{HOX}^{+}$leukemias. In addition, within COSMe-II T-ALLs, the lowest levels of cluster B methylation were also present in immature ETP-ALLs and a HOXA+ T-ALL (Supplementary Fig. S4A).

Altogether, we here show robust COSMe classification as a more elaborate version of the previously established CIMP classification in T-ALL, using different DNA profiling methods and two independent leukemia patient cohorts.

Figure 1. DNA methylation profiling in normal and malignant T cells. A, Unsupervised clustering of mean-centered methylation score ( $\beta$ values) of the 5,000 most variably methylated CpGs in 109 T-ALL cases and 10 subsets of developing thymocytes (two biological replicates each), with indication of the three main clusters of CpG probes and their location with respect to CpG context. T-ALL subtypes and genetic defects of all T-ALL cases are indicated below the heatmap. Mean $\beta$ values of cluster $A(\mathbf{B})$ and cluster $B(\mathbf{C}) C p G$ sites in COSMe type I and II T-ALLs and normal thymic precursors. $\mathrm{DP}, \mathrm{CD}^{+} \mathrm{CD}^{+}$double positive; ISP, immature single positive; SP, single positive. D, Percentage of probes located inside different genomic categories for

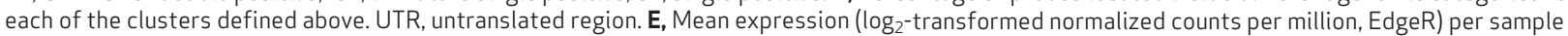
of genes linked to CpG sites in clusters A, B, and C (A) in a T-ALL cohort (GSE110637) plotted separately for TAL1-rearranged T-ALLs (enriched in COSMe-I) and T-ALLs without such rearrangements (enriched in COSMe-II). Normal thymocytes (GSE151079) were included as healthy controls. Genes in the NOTCH1 pathway were used as positive reference value for T-ALL. F, ChIP-seq enrichment in clusters $\mathrm{A}$, B, and C for H3K27me3 in CD34+ $\mathrm{HSPCS}$ (GSM2277181) and in the JURKAT T-ALL cell line (GSM2279072) and for PU.1 in CD34+ HSPCs (GSM1816090) and the TALL-1 cell line transfected with FLAG-PU.1 (GSE128837). Data publicly available from the Gene Expression Omnibus. G, COSMe signature in paired primary-relapsed T-ALL samples (21). Row-scaled $\beta$ values of $C p G$ methylation are shown. 
A

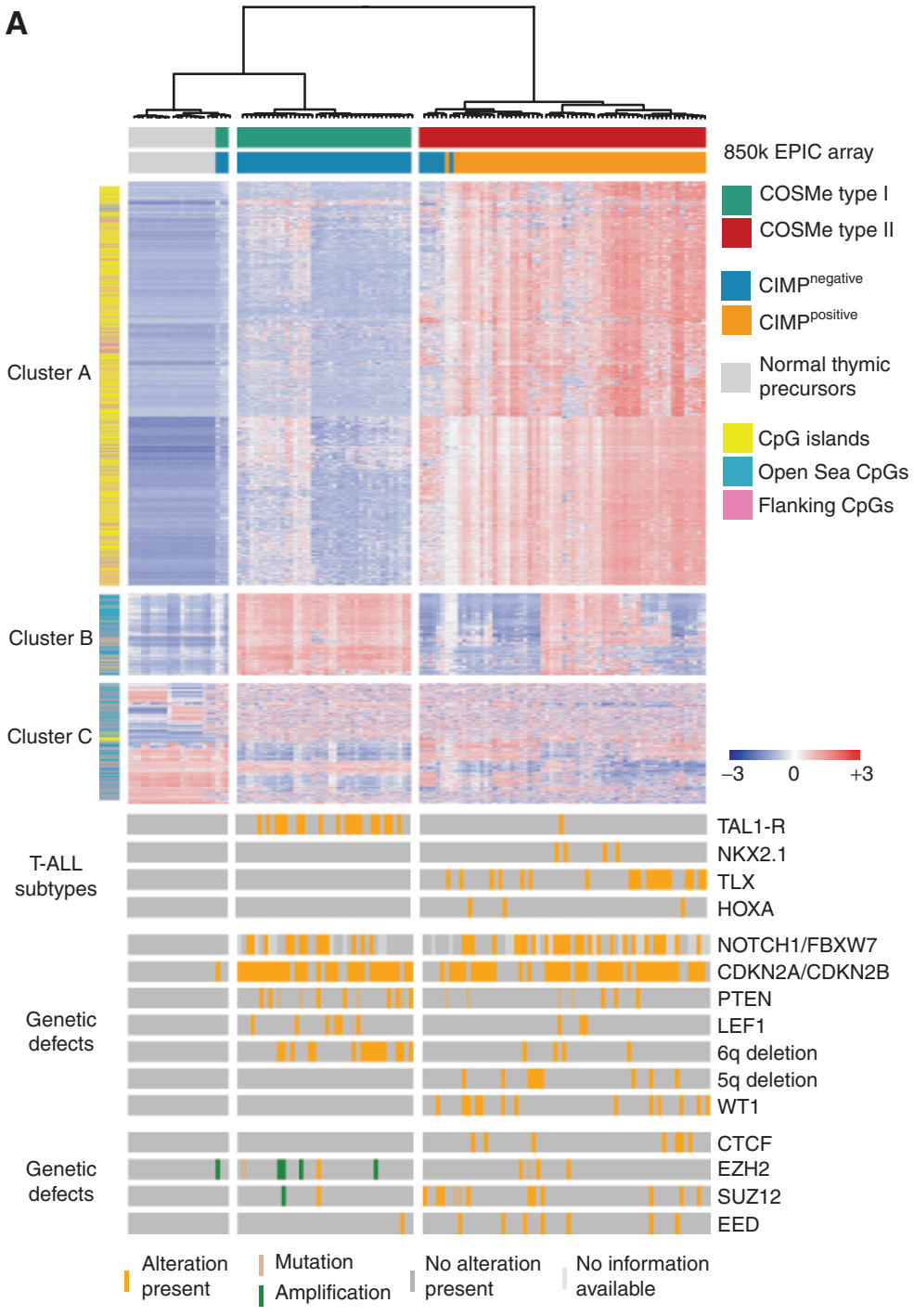

E

Gene expression

Human T-ALLS

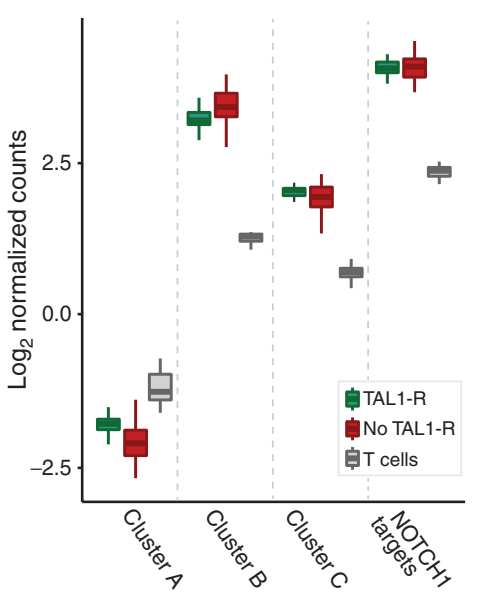

$\mathbf{F}$

H3K27me3 PU.1 HSPCs JURKAT HSPCs TALL-1

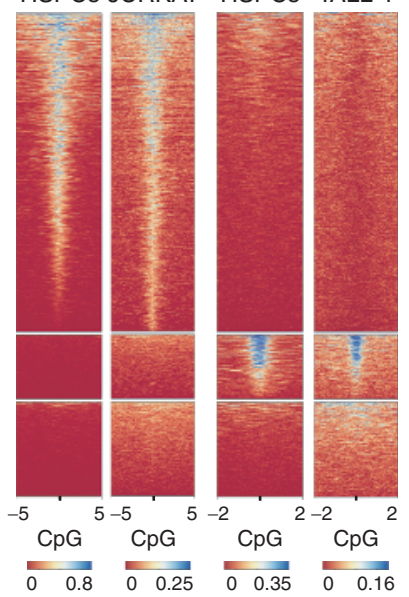

B

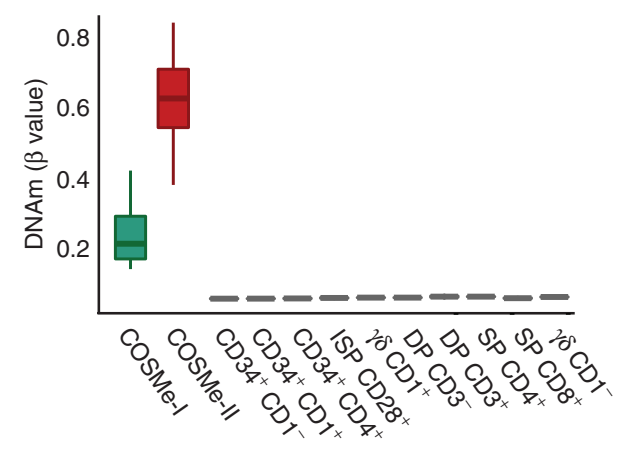

C

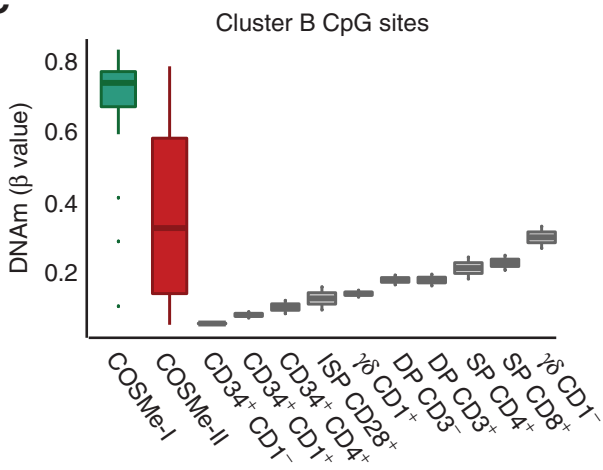

D

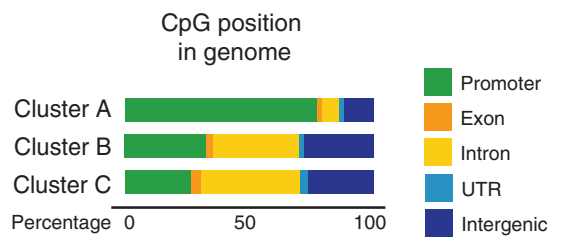

G

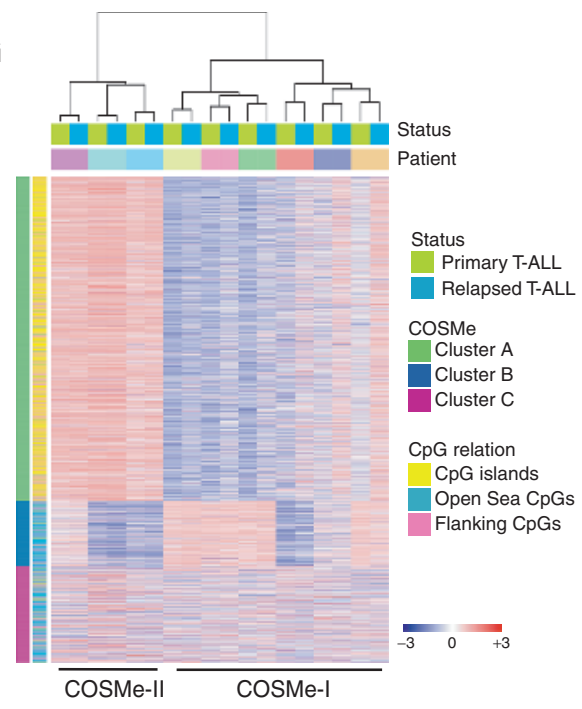




\section{Characterization of CpG Clusters that Define COSMe in T-ALL}

To understand which genes or regulatory pathways are potentially affected by COSMe methylation in T-ALL, we performed enrichment (14) and gene expression analysis $(15,16)$ of transcripts associated with the CpG clusters mentioned above. Of note, cluster A sites were mostly located in gene promoters, while cluster $\mathrm{B}$ and $\mathrm{C}$ CPG sites were mostly located in gene bodies or intergenic regions (Fig. 1D). cluster A CPG sites were enriched for promoters of PRC2 targets (Supplementary Fig. S5), which uniformly showed very low expression in T-ALLs as well as normal thymocytes (Fig. 1E). Using publicly available chromatin immunoprecipitation (ChIP) sequencing (ChIP-seq) data, we confirmed the presence of the repressive $\mathrm{H} 3 \mathrm{~K} 27 \mathrm{me} 3$ histone mark at these cluster A CPG sites in both human $\mathrm{CD} 34^{+}$hematopoietic stem/precursor cells (HSPC) and the T-ALL cell line JURKAT (ref. 17; Fig. 1F).

Notably, cluster B CPG sites did not show enrichment for repressive histone modifications (Supplementary Fig. S5) and were associated with genes higher expressed in T-ALL compared with normal developing $\mathrm{T}$ cells (Fig. 1E). Cluster B sites showed significant enrichment for PU.1 (SPI1) binding motifs (Supplementary Fig. S5), which corresponded to specific binding of PU.1 $(18,19)$ at these exact loci in CD34 ${ }^{+}$ HSPCs and the Flag-tag PU-1-transfected T-ALL cell line TALL-1 (ref. 20; Fig. 1F). Of note, during normal T-cell differentiation, we observed a significant inverse correlation between cluster B PU.1-binding site methylation and SPI1 (which encodes PU.1) expression (Supplementary Fig. S6).

Cluster C CpG sites, characterized by a heterogeneous pattern of DNA methylation, also showed higher expression in T-ALL as compared with normal T cells (Fig. 1E). Cluster C sites showed significant enrichment for genes specifically expressed in T-ALL cell lines, which was not the case for cluster A- or cluster B-associated transcripts (Supplementary Fig. S7A-S7C). More specifically, cluster C sites include CpGs associated with genes known to be involved in T-ALL disease and/or normal T-cell differentiation, such as TLX3, LCK, CDKN2B, BCL2L11, MEF2C, BCL11B, RAG1, RAG2, CD1, CD28, and the TCR loci (Supplementary Table S3). Thus, cluster C $\mathrm{CpG}$ sites are enriched near genes with known roles in normal and malignant T-cell development.

Finally, to evaluate the progression of COSMe methylation from diagnosis to relapse, we investigated paired primary and relapsed T-ALL cases that were previously profiled by EPIC arrays (21). Out of 9 patients analyzed, we found that 6 patients were classified as COSMe-I and 3 patients as COSMe-II at diagnosis (Fig. 1G). Patients at relapse still clustered together with the corresponding primary samples, suggesting that COSMe status is largely conserved from diagnosis to relapse (Fig. 1G). Nevertheless, patients with COSMe-I did show a significant increase in cluster A methylation from diagnosis to relapse (Supplementary Fig. S8).

\section{Reciprocal DNA Methylation and H3K27me3 Association in COSMe-I and COSMe-II T-ALLs}

As described above, COSMe cluster A CPG sites are located in the promoters of PRC2 target genes that uniformly show low expression across all genetic subtypes of human T-ALL. PRC2 is a methyltransferase that primarily produces H3K27me3, a mark of transcriptionally silent chromatin. To validate the enrichment for PRC2 targets at cluster A CpGs, we profiled H3K27me3 in 3 COSMe-I and 3 COSMe-II human T-ALLs using ChIPmentation (22).

Remarkably, at cluster A sites, H3K27me3 was found more abundant in COSMe-I T-ALLs, which have lower levels of DNA methylation at these sites, as compared with COSMe-II T-ALLs (Fig. 2A). To further study this apparent anticorrelation between DNA methylation and H3K27me3, a differential analysis was conducted comparing $\mathrm{H} 3 \mathrm{~K} 27 \mathrm{me} 3$ of COSMe-II with COSMe-I T-ALLs (Fig. 2B). This revealed that the majority of differential regions (88\%) had significantly lower levels of H3K27me3 in COSMe-II compared with COSMe-I T-ALLs (Fig. 2B), as also shown in a genome browser view for the genes ESRRG and DKK2 as representative examples (Supplementary Fig. S9). Genes with differential H3K27me3 were generally low but not differentially expressed between different T-ALL subtypes (Supplementary Fig. S10). Forty-four percent of all genes with differential H3K27me3 in this comparison (Fig. 2B) were also present in cluster $\mathrm{A}$, underlining the reciprocal association between DNA methylation and H3K27me3 at these sites. High levels of cluster A H3K27me3 also corresponded to low levels of cluster A DNA methylation in 4 patients for which paired EPICseq and H3K27me3 profiles were available (Fig. 2C). In line with this, core components of the PRC2 complex showed higher expression in TAL1-rearranged T-ALLs (EZH2, EED, $P_{\text {adj }}<0.05$, SUZ12 not significant; Supplementary Fig. S11), which are most often COSMe-I T-ALLs displaying low levels of cluster A methylation. In contrast, deletions of PRC2 members were significantly more prevalent in COSMe-II T-ALLs, whereas amplifications were exclusively found in COSMe-I T-ALLs (Supplementary Fig. S2).

Finally, from all differential H3K27me3 regions, those that overlapped with CPG islands did show a differential DNA methylation pattern (111 regions, 50\%), whereas the methylation of Open Sea sites was not affected by the presence of differential H3K27me3 (Fig. 2D).

Altogether, these data collectively show a reciprocal association between DNA methylation and H3K27me3 levels at cluster A CPG islands in human T-ALL.

\section{Cluster A CpG Island Methylation Defines the Proliferative History of Human T-ALL}

Cluster A CPG sites are mainly located in promoters of PRC2 target genes that are differentially covered by $\mathrm{H} 3 \mathrm{~K} 27 \mathrm{me} 3$ between COSMe-I and II T-ALL subtypes. Interestingly, DNA hypermethylation at PRC2-enriched $\mathrm{CPG}$ sites has previously been associated with increased age and proliferative history in both hematopoietic stem cells and T-ALL $(11,23)$. Given this, we predicted the mitotic age of our T-ALL patient cohort using the Epigenetic Timer of Cancer (Epitoc; ref. 24) and identified a strong and significant correlation with the level of cluster A methylation, which was not observed for cluster B or cluster C (Fig. 3A).

Furthermore, using the Horvath (25) age predictor, which employs methylation of a set of CpGs to predict a person's actual age in years, an increase in epigenetic age of primary T-ALL samples was observed in comparison with the 
A

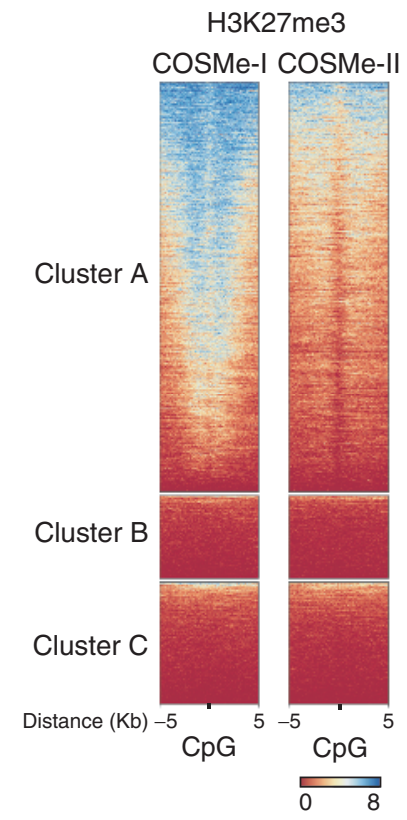

C

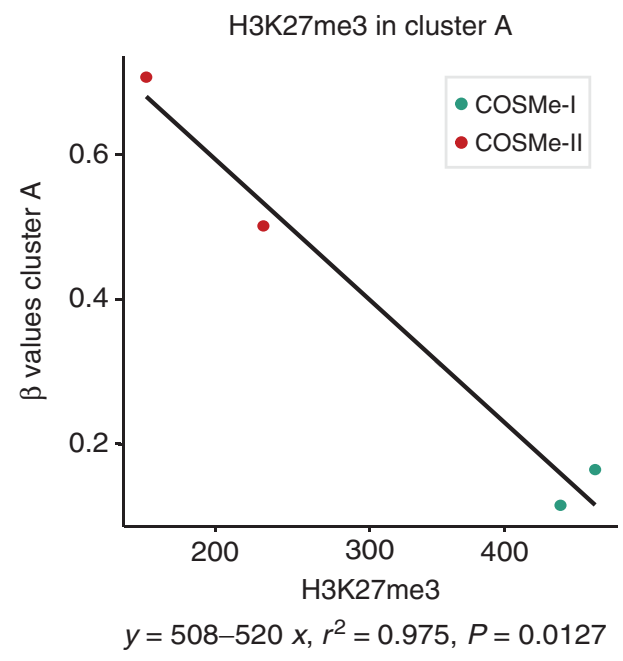

B

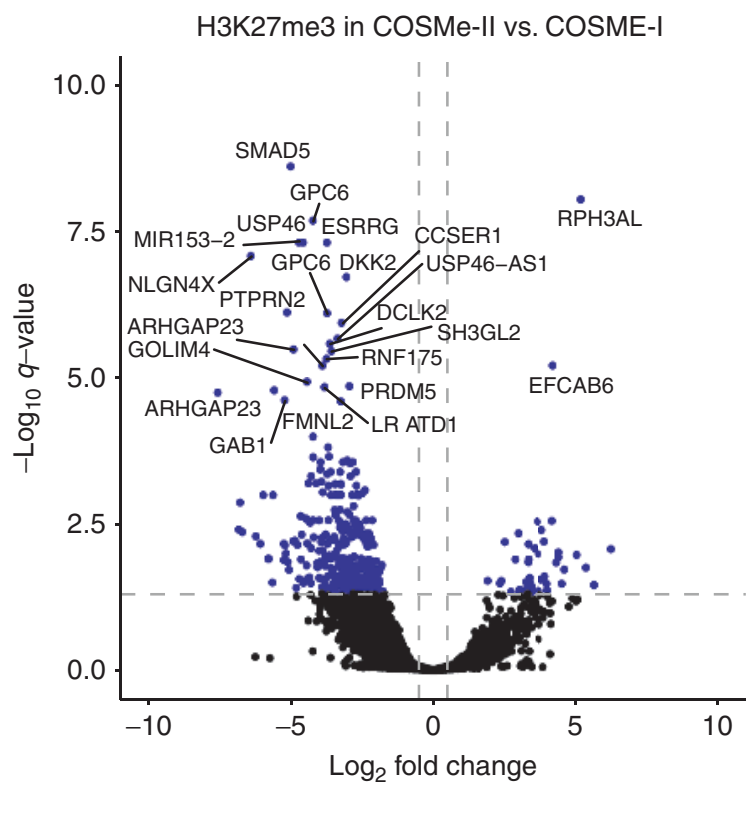

D

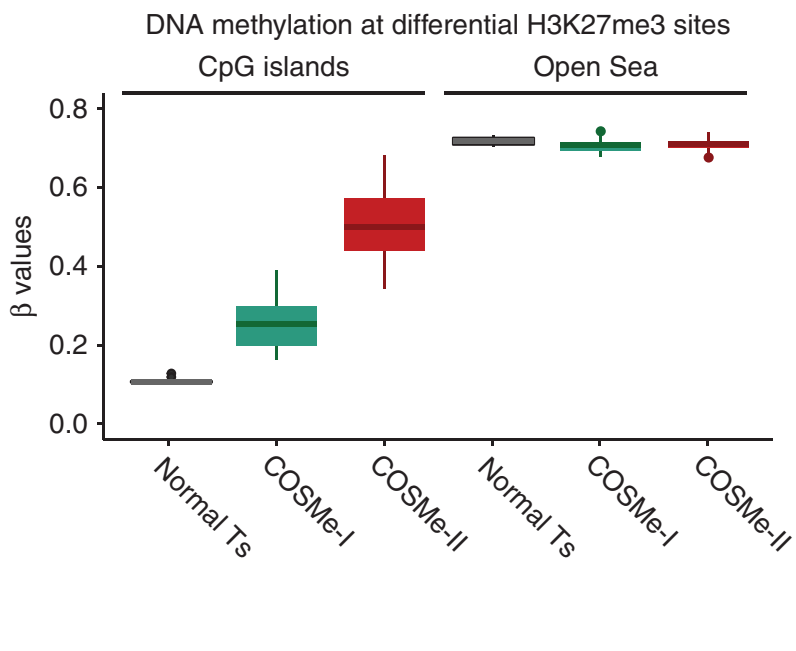

Figure 2. Correlation between cluster A DNA methylation and H3K27me3. A, H3K27me3 binding at cluster A, B, and C sites in COSMe-I and COSMe-II T-ALLs (summarized for visualization, $n=3$ per group). B, Volcano plot of differential H3K27me3 between COSMe-II and COSMe-I T-ALLs. Gene names from the top 25 most differential genes based on $P_{\text {adj }}$ value are indicated. C, Patient-specific comparison between cluster A DNA methylation levels ( $\beta$ values) and corresponding differential H3K27me3 (normalized counts, DESeq2) in COSMe-II versus COSMe-I. Pearson coefficient of the linear correlation with $P$ value is shown. $\mathbf{D}$, The levels of DNA methylation ( $\beta$ values) at all differential H3K27me3 regions between COSMe-II and COSMe-I T-ALLs that were covered by the EPIC array dataset, separately plotted for CpG islands (111 regions) and Open Sea CpGs (111 regions).

actual age at diagnosis (Fig. 3B; Supplementary Table S7). A significant interaction between the patient's age and the Horvath age was indeed observed ( $t$ test, $P<2 \mathrm{e}-16)$. However, although the age at diagnosis was already significantly different between COSMe-I and -II T-ALL patients (Fig. 3C; $P=0.02506)$, the predicted Horvath and Epitoc age showed a much better segregation between the two T-ALL subtypes (Fig. 3B and C; $P<2.2 \mathrm{e}-16$ and 3.85e-16). Thus, cluster A methylation defines the proliferative history of T-ALL, with
COSMe type I having a shorter history of proliferation in comparison with COSMe type II T-ALL.

Proliferation during the time frame from primary diagnosis to relapsed T-ALL disease should also lead to an increase in mitotic age. Indeed, a significant increase in Epitoc age was also observed by comparing 9 paired diagnosis and relapse samples (ref. 21; Fig. 3D). However, the increase in age and methylation was considerably more pronounced in COSMe-I T-ALLs $\left(\Delta^{\text {Epitoc }} 0.11, P=0.0020\right)$ as compared with COSMe-II 
A

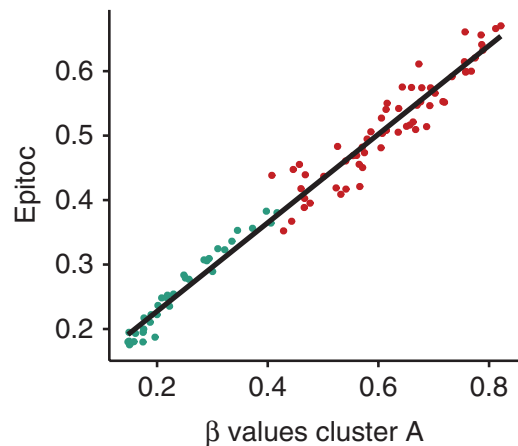

$y=0.093+0.69 x, r^{2}=0.972 P<2.2 e-16$

Cosme type I

cosme type II

B

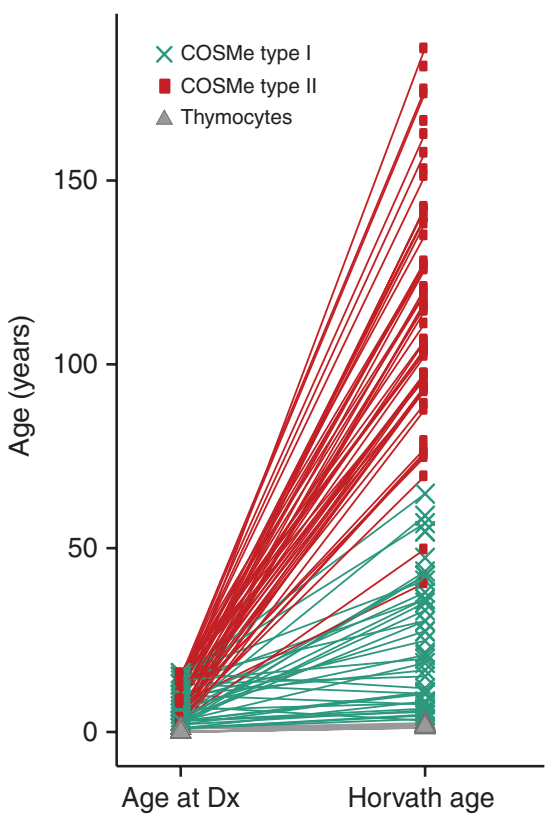

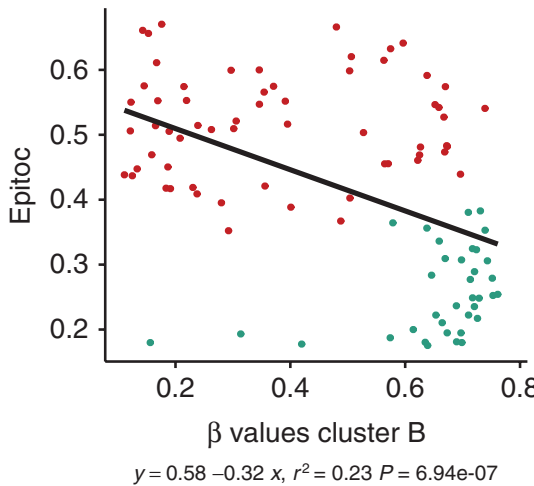

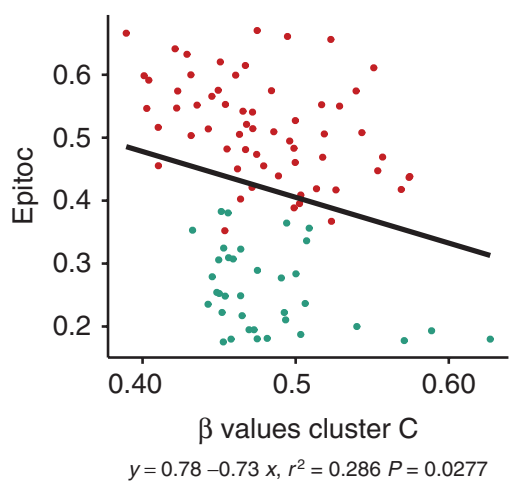

C

\begin{tabular}{c|ccc} 
& Age at Dx & Horvath age & Epitoc age \\
\hline COSMe-I & 6.75 & 24.7 & 0.26 \\
cosMe-II & 8.85 & 112.23 & 0.52 \\
\hline$P$ Value & 0.02506 & $<2.2 \mathrm{e}-16$ & $3.85 \mathrm{e}-16$
\end{tabular}

D T-test, $P=0.003365$

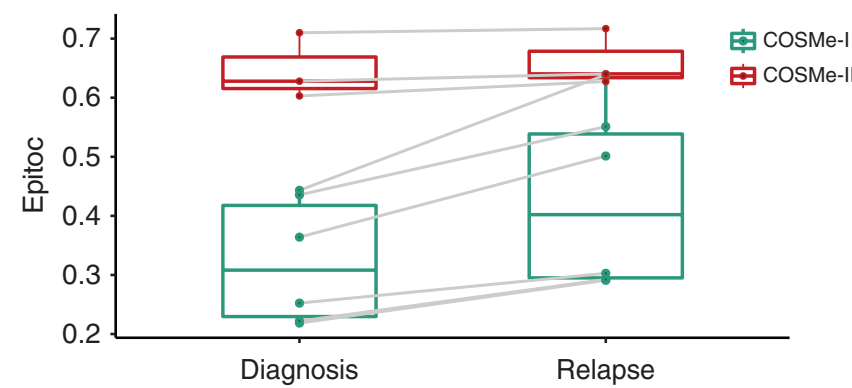

Figure 3. Epigenetic age in T-ALLs at diagnosis and relapse. A, Correlation between epigenetic "Epitoc" age and mean methylation per patient in clusters A, B, and C. B, Age at diagnosis (Dx) and age predicted by Horvath for each patient and normal T-cell subset. C, Mean age at diagnosis (Dx) and in different age predictors in COSMe-I and COSMe-II subgroups with P values of differences (Wilcoxon signed-rank test). D, Epigenetic "Epitoc" age of paired diagnosis-relapse patients.

T-ALLs ( $\left.\Delta^{\text {Epitoc }} 0.015, P=0.0522\right)$, yet there was no significant difference between the time of relapse between both entities (Supplementary Table S8; $P=0.2619$ ). From this limited analysis (21), COSMe-I T-ALLs seem to proliferate at a faster pace than COSMe-II T-ALLs between diagnosis and relapse.

\section{Proliferation of Preleukemic Thymocytes Drives the Aging CpG Island Methylation Signature in T-ALL}

As shown above, cluster A CPG island methylation positively correlates with the proliferative history and mitotic age of human T-ALL cells. To investigate whether two distinct trajectories toward T-ALL development might underlie the observed discrepancy in epigenetic age between COSMe-I and COSMe-II T-ALLs (Fig. 3B and C), we used two known T-ALL mouse models that might recapitulate these features.

First, we investigated whether CD2-Lmo2 transgenic (CD2$L m o 2^{\mathrm{tg}}$ ) mice (26) could function as a model for COSMe-II T-ALLs. This in vivo T-ALL mouse model has a long disease latency and an immature T-ALL phenotype reminiscent of T-ALLs inside the COSMe-II subgroup. In CD2-Lmo2 ${ }^{\text {tg }}$ mice, a long-term self-renewing thymocyte population has been observed many months before tumor development. In contrast to wild-type (WT) control mice, in which the thymus is continuously replenished by progenitor cells from the bone marrow, these preleukemic CD2-Lmo2 ${ }^{\text {tg }}$ thymocytes are 
self-sustaining from young age. Therefore, CD2-Lmo $2^{\text {tg }}$ thymocytes should undergo a gradual aging process in the months prior to malignant transformation.

Second, we investigated whether $L c k-C r e^{\mathrm{tg} /}{ }^{+} \operatorname{ten}^{\mathrm{f} / \mathrm{fl}}$ mice could function as a model for COSMe-I T-ALL. This mouse T-ALL model has a short disease latency and results in the development of more mature murine T-cell leukemias. In addition, PTEN mutations and deletions are also more often observed in human COSMe-I T-ALLs.

To study this, we isolated full thymus from CD2-Lmo2 $2^{\text {tg }}$ and $L c k-C r e^{\mathrm{tg} /+} P t e n^{\mathrm{f} / \mathrm{ll}}$ mice and littermate controls at different time points before and after leukemia development and performed DNA methylation profiling by reduced representation bisulfite sequencing (RRBS; $n=4$ biological replicates per condition). The most variably methylated CPGs between CD2-Lmo2 ${ }^{\mathrm{tg}}$ and $\mathrm{Lck}-\mathrm{Cr} \mathrm{e}^{\mathrm{tg} /+} \mathrm{Pten}^{\mathrm{f} / \mathrm{fl}}$ thymocytes and blasts (Supplementary Table S9) were more often situated in CpG islands (Fig. 4A, cluster 1) than in Open Sea-enriched regions (Fig. 4A, clusters 2 and 3). The CPG methylation in cluster 1 was highly increased in preleukemic and leukemic CD2-Lmo2 ${ }^{\mathrm{tg}}$ mice, but not in $L c k-C r e^{\mathrm{tg} /+}$ Pten $^{\mathrm{f} / \mathrm{fl}}$ mice isolated before leukemia development. In leukemic $L c k-C r e^{\mathrm{tg} /+} \operatorname{Pten}^{\mathrm{f} / \mathrm{fl}}$ mice, cluster 1 methylation was only moderately increased compared with CD2-Lmo2 $2^{\text {tg }}$ samples at the same stage of disease manifestation.

Notably, cluster $1 \mathrm{CpGs}$ were mostly situated in promoter regions (Fig. 4B) of lowly expressed genes (Fig. 4C) and displayed enrichment for PRC2 target genes and H3K27me3 $(27,28)$ at these sites (Supplementary Fig. S12; Fig. 4D), thus showing similarity with the hypermethylation phenotype of T-ALLs in the human cluster A. In contrast, we did not find any evidence in these mouse models for potential overlap between murine clusters 2 or 3 and the human COSMe cluster B.

To further confirm similarities between murine cluster 1 and human cluster A, we subsequently looked at cross-species overlap at the gene level. Notably, 726 of 2,248 CpG sites in mouse cluster 1 overlapped with human gene orthologs in cluster A (overlap significant at $P<0.0001$, exact hypergeometric probability). These sites, which we termed cluster $A^{\mathrm{mm}}$ sites (Supplementary Table S10), were equally significantly enriched for PRC2 target genes $\left(P_{\mathrm{adj}}<0.0001\right)$.

Cluster $\mathrm{A}^{\mathrm{mm}}$ regions showed a gradual increase in methylation with aging in $C D 2-L m o 2^{\text {tg }}$ thymocytes but remained constant in corresponding WT mice from 8, 16, and 24 weeks old (Fig. 4E). Already in 8-week-old CD2-Lmo2 ${ }^{\text {tg }}$ thymocytes, a strong increase in methylation could be observed compared with WT control cells of the same age. In contrast, in Lck$\mathrm{Cre}^{\mathrm{tg} /+}$ Pten $^{\mathrm{f} / \mathrm{fl}}$ mice at 8 weeks, no difference in cluster $\mathrm{A}^{\mathrm{mm}}$ methylation was detected. We found that cluster $A^{\mathrm{mm}}$ methylation did not further increase in fully transformed CD2$L m o 2^{\text {tg }}$ leukemia, sacrificed on average at $35.75 \pm 8.28$ weeks, compared with preleukemic CD2-Lmo2 $2^{\text {tg }}$ at 24 weeks (Fig. 4E), whereas only a moderate increase in cluster $\mathrm{A}^{\mathrm{mm}}$ methylation was detected in leukemic $L c k-C r e^{\mathrm{tg} /+} \operatorname{Pten}^{\mathrm{f} / \mathrm{fl}}$ mice (sacrificed on average at $18 \pm 3.83$ weeks). Finally, the epigenetic age, calculated using the mouse-specific calculation method of Petkovich and colleagues (29), increased over time in preleukemic thymocytes of the CD2-Lmo2 ${ }^{\mathrm{tg}}$ mice, which was not the case in the $L c k-C r e^{\mathrm{tg} /+} \operatorname{Pten}^{\mathrm{f} / \mathrm{fl}}$ model (Fig. 4F).
Thus, preleukemic, self-renewing thymocytes display a murine CPG island DNA hypermethylation signature that recapitulates features of the $\mathrm{CPG}$ island hypermethylation phenotype that we observed in human COSMe-II T-ALL. Therefore, CD2-Lmo2 ${ }^{\text {tg }}$ might serve as a bona fide model for COSMe-II T-ALL development. In contrast, this CpG island hypermethylation phenotype was not observed in preleukemic thymocytes of $\mathrm{Lck}-\mathrm{Cr} \mathrm{e}^{\mathrm{tg} /+} \mathrm{Pten}^{\mathrm{f} / \mathrm{fl}}$ mice, suggesting that this murine model is more similar to COSMe-I human T-ALL. Furthermore, based on these results, COSMe-I and COSMe-II T-ALLs might have followed a different trajectory toward leukemia, marked by the absence or presence of a thymic selfrenewing population that preceded leukemia development.

\section{Age-Related CpG Island Hypermethylation Is Resistant to the FDA-Approved Hypomethylating Agent Decitabine}

DNA hypomethylating agents such as azacitidine and decitabine are approved for myelodysplastic syndrome and acute myeloid leukemia (30). Interestingly, few publications have also investigated the possible use of DNA hypomethylating agents for the treatment of human T-ALL. One ETP-ALL and T-ALL patient responded to decitabine and achieved complete response in a phase I clinical trial (31). In addition, durable remissions have been reported for few other cases of (early) T-cell precursor ALL treated with decitabine as monotherapy (32-34) or in combination with the BCL-2 inhibitor venetoclax $(35,36)$. The largely perturbed DNA methylation profile observed in T-ALL further supports the rationale of using DNA hypomethylating agents for the treatment of this disease. However, the actual mechanism of action that could explain the antileukemic properties of these hypomethylating agents and their putative effect on aberrant DNA methylation in T-ALL, as exemplified by the COSMe phenotype, has remained largely unclear.

To address these questions, we first evaluated the antileukemic properties of decitabine in a preclinical setting using four different primary human T-ALL patient-derived xenograft (PDX) models with variable genetic backgrounds (Supplementary Table S11A-S11H). Notably, 10 days of decitabine (5 days on, 2 days off, $0.5 \mathrm{mg} / \mathrm{kg}$ ) significantly improved leukemia-free survival for all PDX samples analyzed, including a sample that originated from a STIL-TAL $1^{+}$ mature T-ALL at second relapse (Fig. 5A). As expected, this improved survival coincided with a significant decrease in blast percentage in the peripheral blood, as shown for one PDX T-ALL (Supplementary Fig. S13).

We selected two PDX T-ALLs for DNA methylation profiling representative for a COSMe-I and a COSMe-II T-ALL based on the genetics (PDX\#1 and PDX\#2). For this, 7 days after start of the treatment, leukemic blasts from control and decitabinetreated animals $\rightarrow(0.5 \mathrm{mg} / \mathrm{kg}, n=3$ each group $)$ were collected from the spleen. We performed differential methylation analysis comparing decitabine treatment with vehicle control for each PDX in vivo. As expected, a very large number of regions showed a significant decrease in DNA methylation, but a substantial portion of CpGs also displayed an increase in methylation, especially those located in CpG islands (Fig. 5B; DESeq2 $\left.P_{\text {adj }}<0.05\right)$. Despite these global changes in DNA methylation, decitabine did not drastically affect DNA methylation 
A

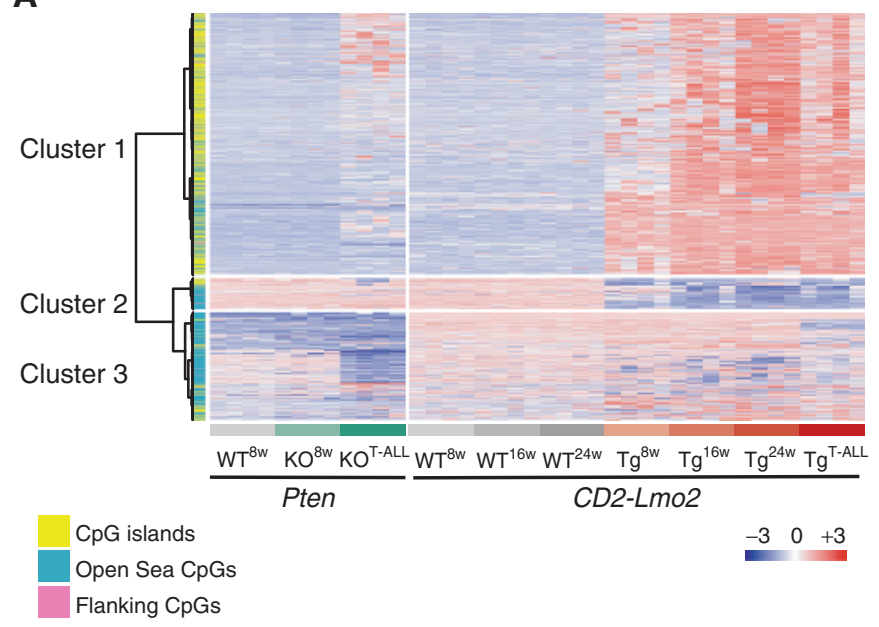

B
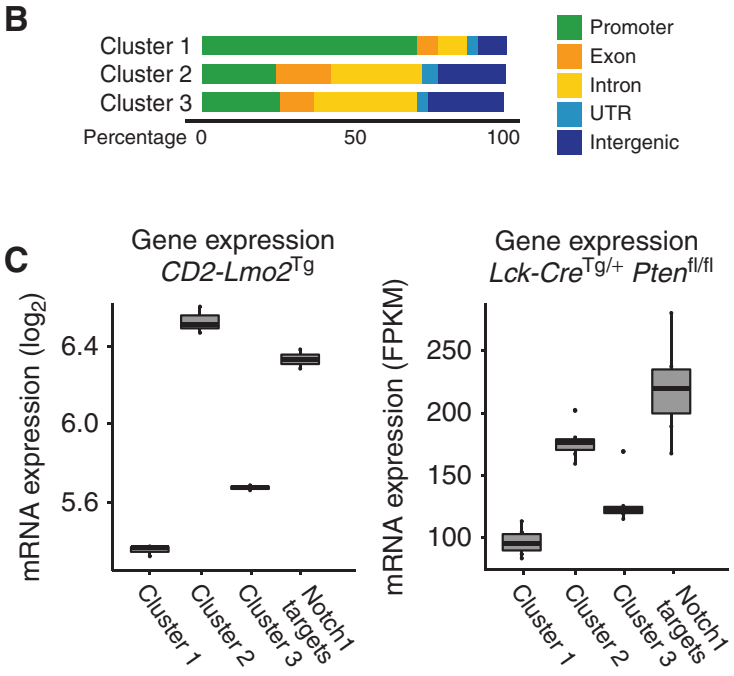

Cluster $\mathrm{A}^{\mathrm{mm}}$ methylation

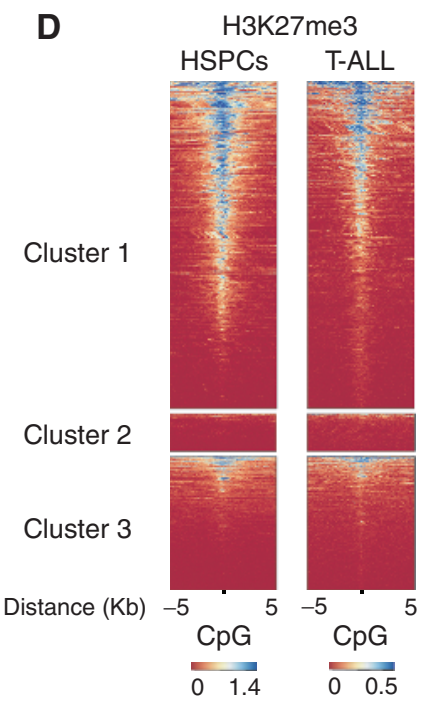

$\mathbf{F}$

Epigenetic age

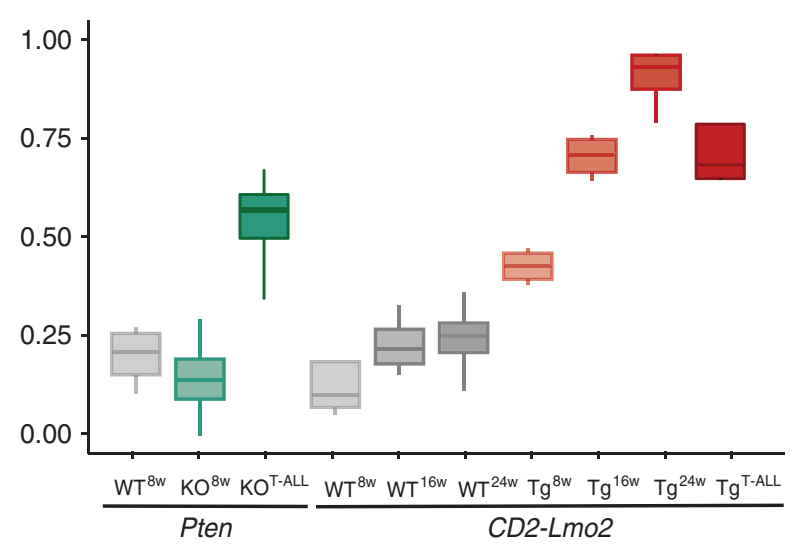

E

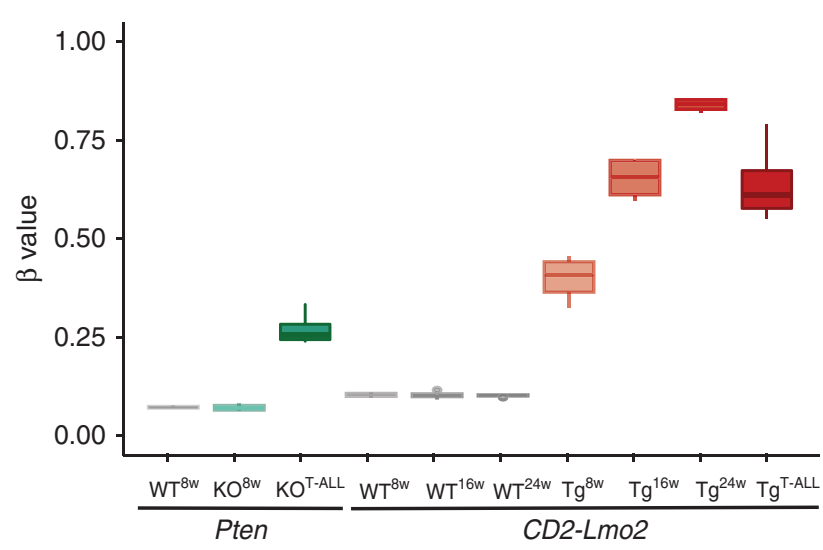

Figure 4. Murine in vivo T-ALL models recapitulate the COSMe phenotype. A, Mean-centered methylation scores ( $\beta$ values) of the most variably methylated CpGs in RRBS profiling of aging $(8,16$, and 24 weeks) preleukemic and fully transformed CD2-Lmo $2^{\text {tg }}$ mice (Tg), 8-week-old and fully transformed $L c k-C r e^{T g /+}+\operatorname{ten}^{\mathrm{fl} / \mathrm{fl}}$ mice (KO), and littermate controls (WT), with indication of three main subclusters and their relation to $\mathrm{CpG}$ context. Data from 4 mice per condition. B, Percentage of $\mathrm{CpGs}$ located in different genomic categories for each of the clusters defined in $\mathbf{A}$. UTR, untranslated region. C, Per sample mean expression of genes in the clusters defined in $\mathbf{A}$ shown in CD2-Lmo $2^{\text {tg }}$ mice (Limma $\log _{2}$ normalized counts, microarray data, GSE49164) or in Lck-Cre $e^{\mathrm{Tg} /+}+\operatorname{ten}^{\mathrm{fl} / \mathrm{fl}}$ (FPKM RNA sequencing, GSE115346). Notch1 pathway genes are shown as positive reference values. D, ChIP-seq of H3K27me 3 in murine clusters 1 to 3 (A) in CD34+ HSPCs (GEO: GSM4067369) and mouse T-ALL (GEO: GSM1506768). E, Methylation at 726 cluster 1 $\mathrm{CpGs}$ (defined above in $\mathbf{A}$ ) that overlap with genes in COSMe cluster $\mathrm{A}$ (defined in Fig. 1A, now termed cluster $\mathrm{A}^{\mathrm{mm}}$ sites) in aging preleukemic and transformed CD2-Lmo $2^{\mathrm{tg}}, \mathrm{Lck}-\mathrm{Cr} e^{\mathrm{Tg} /+}+\mathrm{Pten}^{\mathrm{fl} / \mathrm{fl}}$, and control mice. F, Relative epigenetic age estimation for (pre)leukemic thymocytes from CD2-Lmo2tg, Lck-Cre ${ }^{\mathrm{Tg} /+} \operatorname{Pten}^{\mathrm{fl} / \mathrm{fl}}$, and control mice. 
A

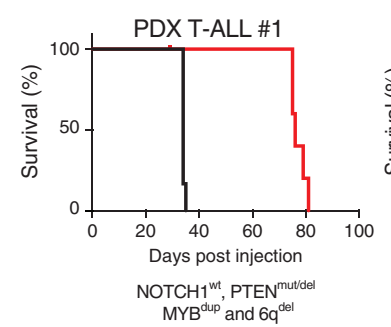

MYB $^{\text {dup }}$ and $6 q^{\text {del }}$

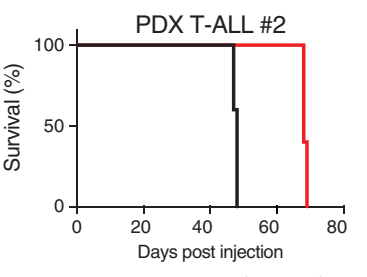

$\mathrm{TLX3}^{+}, \mathrm{NOTCH}^{\text {mut }}$, $\mathrm{PHF}^{\text {mut }}$ CDKN2A/2 ${ }^{\text {del }}$ MYB $^{\text {dup }}$ and CTCF $^{\text {de }}$
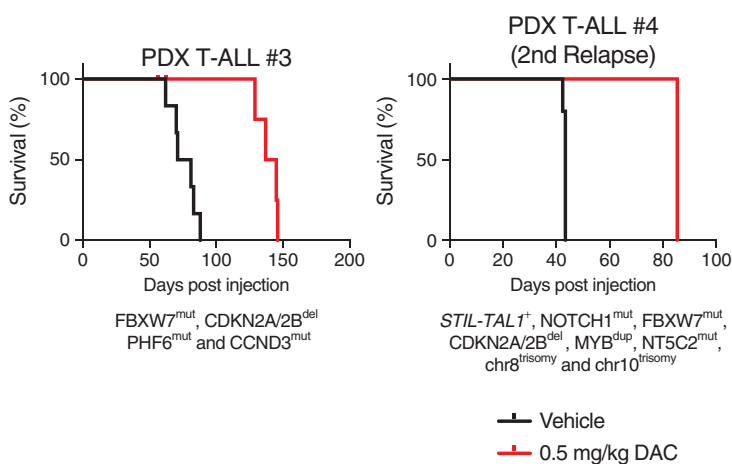

C

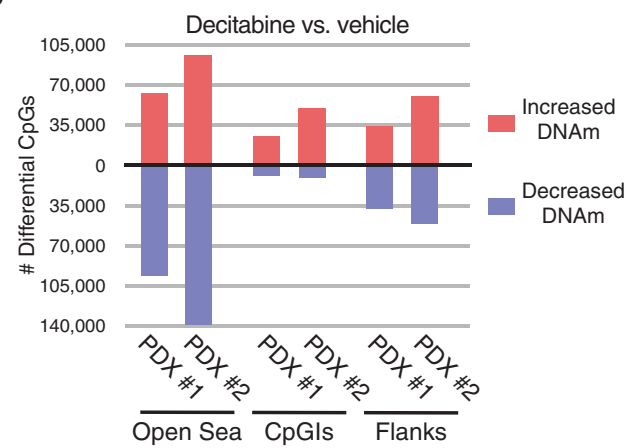

D

Decitabine vs. vehicle

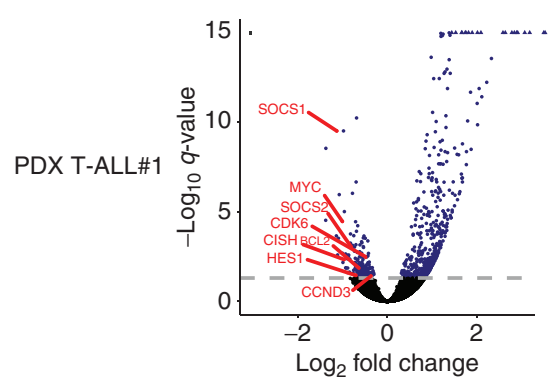

E

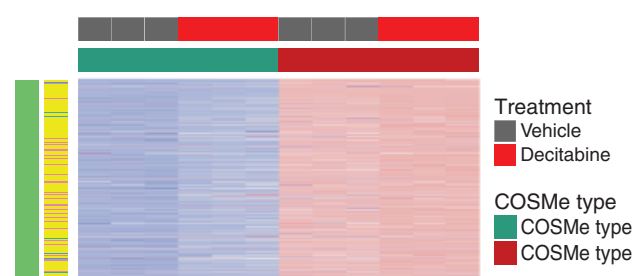

cosme cluster

Cluster A

Cluster B

Cluster C

CpG relation

$\mathrm{CpG}$ islands

Open Sea CpGs

Flanking CpGs

$I^{+3}$

$I^{+3}$

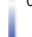

PDX T-ALL\#1 $\quad$ PDX T-ALL\#2

Hallmark MYC targets v1
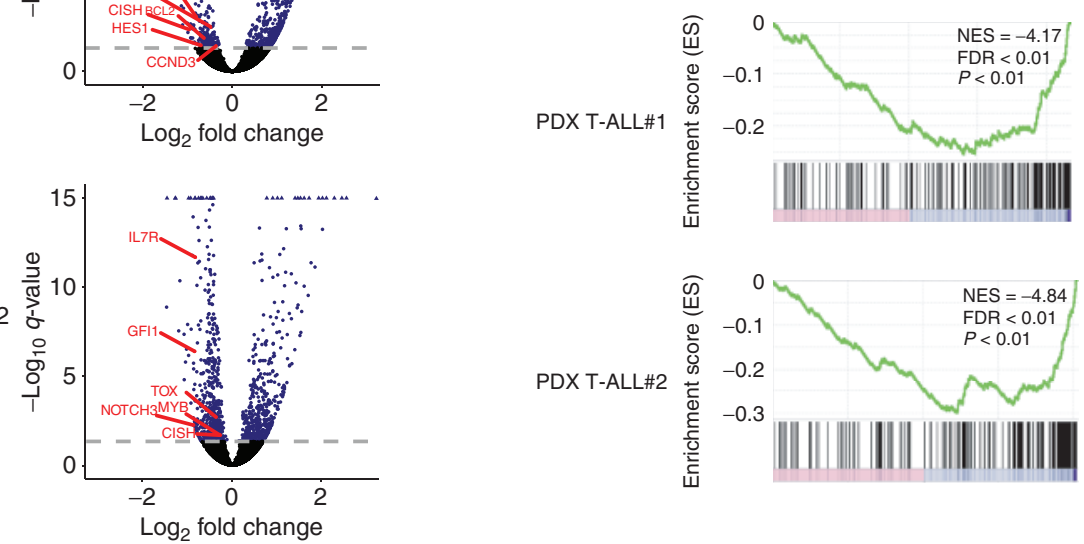

Figure 5. DNA methylation profiling of T-ALL PDXs treated with decitabine in vivo. A, Mice engrafted with T-ALL patient samples were treated two cycles with vehicle or decitabine (DAC, $0.5 \mathrm{mg} / \mathrm{kg}$ body weight) for 5 consecutive days followed by 2 days off and followed for survival analysis. KaplanMeier analysis of leukemia-free survival is shown (log-rank Mantel-Cox test) for each of the 4 PDX samples analyzed. B, Mice engrafted with T-ALL patient samples were treated with vehicle or decitabine $(0.5 \mathrm{mg} / \mathrm{kg}$ body weight) for 5 consecutive days. Seven days after treatment initiation, leukemic blasts from control and decitabine-treated animals ( $n=3$ each group) were collected from the spleen and used for DNA methylation profiling with EPIC arrays. Number of significantly differentially methylated $\mathrm{CpGs}$ is shown for each PDX comparing decitabine-treated with vehicle-treated samples, with respect to their location inside different genomic categories. Red bars indicate more methylation after decitabine treatment, whereas blue bars mark a decrease in methylation upon treatment. CpGI, CpG island; DNAm, DNA methylation. C, $\beta$ values of COSMe CpGs in PDX\#1 and PDX\#2 T-ALLs with decitabine or vehicle treatment. Methylation at the 5,000 COSMe CpG sites was used to determine COSMe-I and COSMe-II T-ALL subtypes. D, RNA-seq of samples described in $\mathbf{A}$. Volcano plots show $\log _{2}$ fold change of protein-coding gene expression upon decitabine treatment for PDX T-ALL\#1 and PDX T-ALL\#2. Genes with $P_{\text {adj }}$ value lower than 0.05 are represented in blue (Deseq2). E, Preranked GSEA of PDX\#1 and PDX\# 2 in decitabine versus control conditions. 
Table 1. Summary overview of DNA methylation clusters in COSMe type-I and COSMe type-II T-ALLs and normal T cells with different characteristics

\begin{tabular}{|c|c|c|c|}
\hline & Normal T cells & COSMe-I T-ALL & COSMe-II T-ALL \\
\hline \multirow[t]{2}{*}{ Cluster A } & No DNA methylation & Low DNA methylation & High DNA methylation \\
\hline & & High H3K27me3 & Low H3K27me3 \\
\hline \multirow[t]{2}{*}{ PRC2 target genes } & & Epigenetically young & Epigenetically old \\
\hline & & Lck-Cre ${ }^{T g /+}$ Pten ${ }^{f l / f l}$ mouse model & CD2-Lmo2'g mouse model \\
\hline Cluster B & $\begin{array}{l}\text { Low DNA methylation increases } \\
\text { with maturation of thymocytes }\end{array}$ & High DNA methylation & $\begin{array}{l}\text { Two subgroups with low or high DNA } \\
\text { methylation }\end{array}$ \\
\hline PU.1 binding sites & $\begin{array}{l}\text { Inversely correlated with PU.1 } \\
\text { expression }\end{array}$ & & $\begin{array}{l}\text { Immature T-ALLs have low cluster } \\
\text { B methylation }\end{array}$ \\
\hline Cluster C & Heterogeneous DNA methylation & Heterogeneous DNA methylation & Heterogeneous DNA methylation \\
\hline Genetics & & $\begin{array}{l}\text { Enriched for TAL1-rearranged } \\
\text { T-ALLs }\end{array}$ & $\begin{array}{l}\text { Enriched for HOXA, TLX3, and } \\
\text { NKX2-1 T-ALLs }\end{array}$ \\
\hline
\end{tabular}

in the 5,000 human COSMe CpGs, neither in PDX\#1, which we determined to be a COSMe-type I cluster $\mathrm{B}^{+} \mathrm{T}$-ALL, nor in the COSMe type II cluster $\mathrm{B}^{+} \mathrm{PDX} \# 2$ (Fig. 5C).

To obtain additional insights in the mechanism of action of hypomethylating agents in T-ALL, we used the same samples (PDX T-ALL\#1 and \#2, 5 days, $0.5 \mathrm{mg} / \mathrm{kg}, n=3$ each group) for RNA sequencing (RNA-seq). Decitabine treatment resulted in significant differential expression of 456 (PDX T-ALL\#1) and 886 (PDX T-ALL\#2) protein-coding genes (Deseq2, $P_{\text {adj }}<0.05$; Supplementary Table S12A and S12B). Of these differentially expressed genes, 361 (PDX\#1) and 348 (PDX\#2) genes also showed a significant decrease in methylation at one or more CpGs (Supplementary Table S13A and $\mathrm{S} 13 \mathrm{~B})$. However, the transcriptome is less disturbed than one would expect, as decitabine induced profound genome-wide DNA hypomethylation, which globally followed CPG density (Supplementary Fig. S14A and S14B). The genes downregulated by decitabine more frequently had $\mathrm{CPG}$ islands in their promoters $(57 \%)$ compared with upregulated or randomly selected genes (both $23 \%, \chi^{2}$ test, $P<0.00001$ ).

As decitabine did not revert the COSMe methylation signature, we further looked at the gene expression changes induced by this hypomethylating agent to explain its antileukemic properties. Both PDX samples showed significant upregulation of tumor suppressor genes (TSG) upon decitabine treatment, including BCL2L11 (BIM), BBC3 (PUMA), and $B M F$ (Supplementary Table S12A and S12B). In addition, multiple known T-ALL oncogenes were significantly downregulated by in vivo decitabine treatment, including $M Y C$, HES1, and BCL2 in PDX\#1 and NOTCH3, GFI1, IL7R, CISH, $M Y B$, and TOX in PDX\#2 (Fig. 5D). Enrichment analysis revealed that decitabine induced a global downregulation of MYC target genes in both PDX samples, as exemplified by preranked gene set enrichment analysis (GSEA; ref. 37; Fig. 5E; Supplementary Table S14). To further validate these findings, we performed additional RNA-seq on five human T-ALL cell lines (LOUCY, PER117, PEER, MOLT16, and TALL-1) following in vitro decitabine treatment $(1 \mu \mathrm{mol} / \mathrm{L}, 48$ hours). The differentially expressed genes from both PDX samples significantly overlapped with the differential data from the cell lines (33\% in PDX\#1 and 35\% in PDX\#2). Indeed, also in these cell lines, significant upregulation of TSGs like $B M F$ and $B B C 3$ (PUMA) and coordinate downregulation of the MYC pathway upon treatment with decitabine were observed (Supplementary Fig. S15A and S15B; Supplementary Table S15A-S15F).

Several studies showed that DNA methylation can alter the binding of CTCF to influence the three-dimensional architecture of the genome (38-40); therefore, we compared the genes downregulated by decitabine in both PDX and T-ALL cell lines with gene expression in CTCF-depleted acute leukemia (41). Interestingly, we observed a very significant enrichment for downregulated genes in both datasets by GSEA (Supplementary Fig. S16), providing a potential mechanism for decitabine-induced downregulation of the MYC pathway in human T-ALL.

\section{DISCUSSION}

In this study, we directly compared the genome-wide DNA methylation landscape in human T-ALL with human thymocytes covering the complete trajectory of normal T-cell development. This simultaneous DNA methylome analysis of T-cell leukemias with their putative cell of origin empowered us to distinguish cell-of-origin methylation profiles from leukemia-specific alterations at CPG islands and Open Sea sites. Integration of DNA methylation profiles with gene expression signatures and ChIP-seq allowed us to obtain novel insights in the functional relevance of differentially methylated CPG sites between normal and malignant $\mathrm{T}$ cells, and to distinguish two trajectories toward T-ALL development.

We built a DNA methylation-based signature for T-ALL, termed COSMe. COSMe comprises three clusters that can divide T-ALLs in two subgroups, COSMe type I and COSMe type II, which both have unique characteristics, as summarized in Table 1. First, COSMe cluster A sites were closely related to the $\mathrm{CPG}$ sites that have previously been used to determine CIMP status in T-ALL (42) and showed low expression in both normal developing thymocytes and human T-ALLs. An inverse correlation between cluster A DNA methylation and H3K27me3 was observed. This interplay could explain the low expression of cluster A genes across all genetic subtypes of human T-ALLs, as repression 
is maintained by different mechanisms in COSMe-I (PRC2-mediated repression) and COSMe-II T-ALLs (DNA methylation-mediated repression). This phenomenon has previously been observed in murine Ezh2 knockout ETPALL (43) that displayed increased DNA methylation. In ETP-ALL, EZH2 inactivating events were previously linked to oncogenic active stem and progenitor cell genes (44). Also, in our cohort, inactivating alterations targeting PRC2 members were more prevalent in COSMe-II T-ALL. However, the relation between PRC2 and DNA methylation is complex, as PRC2 has been shown to recruit both DNMT (45) and TET enzymes (46). Therefore, PRC2 might potentially affect both methylation as well as demethylation, depending on the cellular context. However, the mechanism and functional relevance of these different levels of H3K27me3 between human COSMe-I and COSMe-II T-ALLs remains to be established. In addition, additional studies will be required to further unravel how these epigenetic alterations exactly create a permissive landscape for T-ALL transformation or if they are just the result of specific cell-intrinsic antitumor mechanisms.

Next, our study linked DNA hypermethylation in COSMe cluster A to the proliferative history of the cancer cells, thereby confirming (11) that COSMe-II T-ALLs are epigenetically older and displayed a longer mitotic history as compared with COSMe-I T-ALL. In line with this, we showed that relapsed COSMe-I T-ALLs displayed a larger increase in epigenetic age during their progression from diagnosis to relapsed disease. The younger mitotic age in combination with a faster rate of cellular proliferation might potentially contribute to the more aggressive nature of COSMe-I T-ALLs as compared with COSMe-II leukemias.

Furthermore, we could recapitulate these methylation features distinguishing fast and aggressive COSMe-I from the slower-transforming COSMe-II T-ALLs in two distinct T-ALL mouse models: the spontaneous CD2-Lmo2 ${ }^{\text {tg }} \mathrm{T}$-ALL mouse model, which mimics immature T-ALL development and has a long disease latency, and the fast-transforming Lck-Cre $e^{\mathrm{tg} /+}$ $P t e n^{\mathrm{f} / \mathrm{fl}}$ mouse model, as a model of more mature human T-ALL, where PTEN deletions are more abundant. Notably, these findings establish $L c k-C r e^{\mathrm{tg} /+} P t e n^{\mathrm{f} / \mathrm{ll}}$ and CD2-Lmo2 ${ }^{\mathrm{tg}}$ as models to further study COSMe-I and COSMe-II T-ALL cluster A methylation in vivo, but also provide evidence that a preleukemic self-renewing thymocyte population might also exist in human COSMe-II T-ALL. Indeed, in CD2-Lmo $2^{\text {tg }}$ mice, but not in $L c k-C r e^{\mathrm{tg} /+} \operatorname{Pten}^{\mathrm{f} / \mathrm{fl}}$ mice, a preleukemic state has been described in which thymic precursors gain self-renewing potential prior to full malignant T-cell transformation. The preleukemic cells gain DNA methylation at these sites before transformation from 8 to 24 weeks, which was not observed in the corresponding aging thymocytes of $L c k-C r e^{\mathrm{tg} /+} \operatorname{Pten}^{\mathrm{f} / \mathrm{f}}$ mice or littermate controls. The methylation at these sites did not further increase after full transformation of the preleukemic cells, suggesting that aberrant methylation at these sites is mainly derived from the preleukemic history of these tumor cells.

Besides cluster A CPG island methylation as a surrogate marker for the epigenetic age and replicative history of tumor cells, cluster B sites enabled additional classification of COSMe type-II T-ALL into two categories based on the methylation level of specific Open Sea-enriched CPG sites. Interestingly, these cluster $\mathrm{B}$ loci displayed significant enrichment for the binding motif of PU.1, a transcription factor critically involved in early T-cell development (47).

Of note, we could show that these cluster B sites are able to distinguish immature from more mature T-ALLs within the COSMe-II leukemias. Furthermore, immunophenotypically validated ETP-ALLs, which are derived from the most immature T-cell precursors, also showed the lowest levels of cluster $\mathrm{B}$ methylation in the validation cohort. Whereas conclusive immunophenotypic data were missing to accurately define ETP-ALLs in the initial T-ALL cohort, these data do collectively suggest that both immature T-ALL and ETP-ALL could be distinguished from other T-ALLs based on the absence of cluster B methylation within COSMe-II leukemias.

Notably, a similar correlation between PU.1-binding site methylation and SPI1 expression was recently also identified in TCF7-SPI1 fusion-positive T-ALLs (4). However, the lack of cluster B methylation in this very aggressive subtype of PU.1-rearranged human T-ALL (4) is most probably caused by the aberrant SPI1 expression downstream of the fusion proto-oncogene rather than being associated with its cell of origin.

Finally, cluster C CpG sites were also enriched for Open Sea sites but showed more heterogeneity in DNA methylation between T-ALLs. Cluster C-associated transcripts showed generally lower expression in normal T-cell subsets but were clearly active in at least some T-ALL tumors and included genes involved in T-ALL disease biology (48) as well as normal T-cell differentiation. Thus, the methylation pattern at some of these cluster C Open Sea CPG sites might be T-ALL specific and potentially hold some information on the genetic abnormalities present in each individual T-ALL.

In the last part of our work, we looked for further evidence for DNA hypomethylating agents as a promising therapeutic strategy for human T-ALL. Indeed, some case reports have previously shown that the FDA-approved drug decitabine might be effective as salvage therapy for T-ALL (31) and ETP-ALL (32-34). In line with this, decitabine here displayed profound antileukemic properties in PDXs from a variety of different human T-ALLs obtained from both primary as well as relapse tumor material; however, and most unexpectedly, these antitumoral effects, which were shown to be mediated by downregulating oncogenic MYC signaling, were observed in both COSMe-I and COSMe-II samples. Indeed, decitabine did not revert the age-related human $\mathrm{CpG}$ island hypermethylation phenotype in vivo. Instead, this hypomethylating agent triggered a profound and genome-wide hypomethylation effect on CpGs located in Open Sea areas, and surprisingly also hypermethylation in $\mathrm{CPG}$ islands. The widely altered DNA methylation profile has only limited effects on the transcriptome of these cells. Decitabine-downregulated genes are reconciled by CTCF depletion, thus DNA hypomethylating agents might alter the binding capacity of CTCF as a potential mechanism to induce changes at the transcriptomic level. Several studies also show a direct link between MYC and DNMTs (49). MYC inactivation has been shown to reduce the expression of DNMT3B in T-ALL (50), and reciprocally, reduced expression of $D N M T 3 B$ resulted in reduced proliferation and tumor maintenance, reflecting the effects observed 
in our decitabine-treated PDX T-ALLs. Thus, downregulation of MYC or inhibition of DNMTs by decitabine might converge on counteracting T-ALL disease burden.

Altogether, our work identifies aging of preleukemic thymocytes as a driver of the CIMP in human T-ALL, revealing different trajectories toward T-cell transformation. Our work provides evidence for the involvement of preleukemic thymocytes in the pathogenesis of human T-ALL, which has previously only been reported in mouse T-ALL models so far. In addition, we provide a biological explanation for the profound differences in epigenetic age between COSMe-I and COSMe-II T-ALLs and show that the FDA-approved hypomethylation drug decitabine shows a promising increase in survival of both epigenetically young and old T-ALLs, but fails to revert age-related $\mathrm{CPG}$ island hypermethylation in human T-ALL xenografts. This very extensive DNA methylome dataset of murine and human T-ALL will be of importance to further increase our understanding of T-ALL disease biology, which could ultimately result in better treatment stratification and the development of novel and less toxic therapeutic strategies for the treatment of this aggressive hematologic malignancy.

\section{METHODS}

\section{Patient Samples, Normal T Cells, and Cell Lines}

DNA from 109 T-ALLs was collected from the previously characterized $(8,51)$ ALL IC-BFM 2002/2009 protocol. Exome sequencing was performed by Novogene. Clinical characteristics of patients treated according to both treatment regimens were not significantly different $\left(\chi^{2}\right.$ test).

Thymocytes were isolated from postnatal thymus suspension of two donors each, as described previously $(9,16)$.

Cell lines were purchased from DSMZ and cultured in RPMI1640 medium (Life Technologies) supplemented with $10 \%$ or $20 \%$ FCS, $100 \mathrm{U} / \mathrm{mL}$ penicillin, $100 \mu \mathrm{g} / \mathrm{mL}$ streptomycin (Life Technologies), and $2 \mathrm{mmol} / \mathrm{L}$ L-glutamine (Life Technologies) at $37^{\circ} \mathrm{C}$ with $5 \%$ $\mathrm{CO}_{2}$. CUTTL-1 and PER-117 were a kind gift from Adolfo Ferrando (Columbia University, New York, NY) and Rishi Kotecha (Telethon Kids Cancer Center, Perth, Western Australia), respectively. Cell lines were screened monthly for Mycoplasma contamination and were consistently negative.

All human samples were acquired with written informed consent according to the Declaration of Helsinki, and the studies were approved by the ethical committee review board of the Department of Pediatric Hemato-Oncology at Ghent University Hospital (Ghent, Belgium).

\section{DNA Methylation Profiling}

Human DNA methylation analysis of 109 T-ALLs was done with the Infinium HumanMethylationEPIC BeadChip array (Illumina). DNA (250 ng) was used for bisulfite conversion by the EZ DNA Methylation Kit (Zymo Research).

DNA methylation profiling of the 14 human T-ALL validation cohort was done by TruSeq Methyl Capture EPIC-seq using $500 \mathrm{ng}$ of DNA. Sequencing was done on the Hiseq3000 (PE150). Reads were trimmed using TrimGalore (v0.4.5) and aligned with Bismark (ref. 52; v0.20.0) on hg38.

RRBS was done on thymus isolated from CD2-Lmo2 $2^{\mathrm{tg}}$ and $L c k-C r e^{\mathrm{tg} /} /$ Pten $^{\mathrm{t} / \mathrm{l}}$ mice and littermate controls ( $n=4$ mice per condition). DNA was isolated from full thymus using the QIAamp DNA Mini Kit (Qiagen). RRBS was done using MSPI digest. Bisulfite-converted DNA libraries were sequenced on Illumina NextSeq500 using the NextSeq 500/550
High Output v2 kit (SE75). Reads were trimmed using TrimGalore (v0.4.5) with -rrbs and -nondirectional and aligned with Bismark (ref. 52 ; v0.20.0) on GRCm38.

Illumina EPIC array and RRBS data were analyzed using RnBeads. hg19 or RnBeads.mm10, respectively (53) in R (versions >3.4). Beta values were obtained after filtering and normalization using the default preprocessing pipeline. EPIC-seq was analyzed using the package "methylkit" in R. Annotation of CpGs to the closest gene was done by ChipPeakAnno (54). Copy number variations were called from EPIC array data using the R packages "minfi" and "conumee."

\section{H3K27me3 Profiling}

Fifty to $100,000 \mathrm{CD}^{+} 5^{+}$sorted cells were fixed using $1 \%$ formaldehyde (Thermo Fisher Scientific 28906) and quenched by glycin $(125 \mathrm{mmol} / \mathrm{L}$ final). Next, cell pellets were lysed in $100 \mu \mathrm{L}$ of short-term complete lysis buffer $[50 \mathrm{mmol} / \mathrm{L}$ Tris- $\mathrm{HCl} \mathrm{pH}$ 8.0, 10 $\mathrm{mmol} / \mathrm{L}$ EDTA, $0.25 \% \mathrm{SDS}, 20 \mathrm{mmol} / \mathrm{L} \mathrm{NaBu}$ histone deacetylase inhibitor, $1 \mathrm{X}$ complete protease inhibitors cocktail EDTA free (Roche, 5056489001)]. Chromatin was sheared on the Bioruptor Pico (Diagenode) using a 15 seconds-on/30 seconds-off, 7-cycle regimen. Sheared chromatin was magnetically immunoprecipitated and tagmentated using the Auto ChIPmentation Kit for Histones (Diagenode, C01011010) on the IP-Star Compact Automated System (Diagenode, B03000002), according to the manufacturer's instructions. Input DNA was decrosslinked, purified (MinElute, Qiagen), and tagmentated (Nextera DNA Library Prep, Illumina). Stripping, end repair, and library amplification were performed according to the ChIPmentation Kit guidelines. Libraries were sequenced with the NextSeq500 (SR75, High Output). Reads were trimmed by Trimmomatic and aligned to hg38 with Bowtie 2 using the parameters - $\mathrm{N} 1$ $-k$ 1. Peaks were called with MACS2 with the respective input control for each patient sample.

\section{Gene Expression Profiling}

Total RNA was isolated using the miRNeasy Mini Kit (Qiagen) and evaluated on the Agilent 2100 bioanalyzer (Agilent Technologies). Library preparation was performed using QuantSeq 3'mRNA-Seq FWD for Illumina (LEXOGEN). cDNA libraries were sequenced as described above. Reads were aligned to GRCh38 using STAR2.4.2a (55) and quantified on Gencode v24.

For visualization of RNA-seq data, EdgeR $\log _{2}$-transformed normalized counts per million were plotted unless mentioned otherwise.

Microarray data normalized with Limma and was $\log _{2}$ transformed.

\section{In Vivo Treatment of Xenografts}

PDXs were established in female NOD/SCID $\gamma$ (NSG) mice. For the initial PDX experiments, upon disease establishment, human leukemic cells were isolated from the spleen. Secondary injections were performed in randomized NSG mice (two groups of 5) and treated two cycles ( 5 days on, 2 days off) with vehicle-only or decitabine $(0.5 \mathrm{mg} / \mathrm{kg}$ body weight).

For RNA and DNA collection, mice were randomized in two groups of 3 and treated for 5 days with vehicle only or decitabine $(0.5 \mathrm{mg} / \mathrm{kg}$ bodyweight). At day 7, animals were sacrificed and tumor cells collected from the spleen.

The animal welfare ethical committee (Ghent University Hospital) approved all animal experiments.

\section{CIMP Classification}

CIMP CPGs (1,099) were defined by filtering (3) CIMP probes to exclude CPGs within five base pairs from European SNPs, crosshybridizing probes, repeated regions (56), and methylation quantitative trait locus (57). Missing values were imputed using K nearest neighbor. 


\section{COSMe Classification}

Five-thousand human COSMe CpGs were defined by most variably methylated CpGs in all normal thymocyte subsets and 109 T-ALLs. The classification of diagnosis and relapsed T-ALLs was obtained by hierarchical clustering of these 5,000 CpGs with Euclidean distance measures and clustering method "ward.D" using row scaling.

\section{Epigenetic Age Calculations}

The Horvath (25) and Epitoc (24) age were calculated with "cgageR." Epigenetic age in mouse samples was estimated by taking the weighted average of CPGs within the $90 \mathrm{CpG}$ age classifier defined by Petkovich and colleagues (29). Only CpGs that had enough coverage $(>5)$ after data imputation with BoostMe (58) were retained. The weighted average was scaled and used as a relative measure of epigenetic age.

\section{Data Analysis and Statistics}

R or GraphPad Prism 6.0 was used for statistical analyses. When applicable, normality was tested using a Shapiro-Wilk test.

Publicly available ChIP-seq data were retrieved from the ChIP-Atlas (59). Heatmaps of ChIPseq data were generated by deepTools (60) computeMatrix, and plotHeatmap with the options reference-point and missingDataAsZero.

Enrichment analyses were performed with GSEA $(37,61)$, DAVID $(62,63)$, and Enrichr $(14,64)$. Hierarchical clustering and heatmaps were generated with R "pheatmap" using row scaling. Differential expression was identified by DeSEQ2 (65).

\section{Data Availability}

All generated data was deposited in NCBI Gene Expression Omnibus (GEO) under accession number GSE155339.

\section{Disclosure of Potential Conflicts of Interest}

T. Lammens reports grants from vzw Kinderkankerfonds during the conduct of the study. D. Deforce reports grants from FWO during the conduct of the study. A.E. Kulozik reports grants and personal fees from Bluebird Bio outside the submitted work. D.J. Curtis reports grants from NHMRC during the conduct of the study. T. Taghon reports grants from Research Foundation - Flanders (FWO), Stichting Tegen Kanker, and Cancer Research Institute Ghent during the conduct of the study. M. Dawidowska reports grants from National Centre for Research and Development Poland (STRATEGMED3/304586/5/ $\mathrm{NCBR} / 2017$ ) during the conduct of the study. No potential conflicts of interest were disclosed by the other authors.

\section{Authors' Contributions}

J. Roels: Conceptualization, data curation, software, supervision, validation, investigation, writing-original draft, writing-review and editing. M. Thénoz: Conceptualization, data curation, formal analysis, writing-original draft, writing-review and editing. B. Szarzyńska: Resources, writing-review and editing. M. Landfors: Resources, software, writing-review and editing. S. De Coninck: Resources, data curation, formal analysis, validation, writing-review and editing. L. Demoen: Resources, formal analysis, validation, writing-review and editing. L. Provez: Resources, formal analysis, methodology, writingreview and editing. A. Kuchmiy: Formal analysis, methodology, writingreview and editing. S. Strubbe: Data curation, formal analysis, investigation, writing-review and editing. L. Reunes: Formal analysis, validation. T. Pieters: Formal analysis, validation, writing-review and editing. F. Matthijssens: Formal analysis, validation, investigation, writing-review and editing. W. Van Loocke: Data curation, software, formal analysis, writing-review and editing. B. ErarslanUysal: Resources, writing-review and editing. P. Richter-Pechańska: Resources, writing-review and editing. K. Declerck: Data curation, software, formal analysis, writing-review and editing. T. Lammens: Resources, writing-review and editing. B. De Moerloose: Resources, writing-review and editing. D. Deforce: Resources, data curation. F. Van Nieuwerburgh: Resources, data curation. L.C. Cheung: Resources, writing-review and editing. R.S. Kotecha: Resources, writingreview and editing. M.R. Mansour: Resources, formal analysis, writing-review and editing. B. Ghesquière: Resources, methodology. G. Van Camp: Resources, methodology. W. Vanden Berghe: Resources, data curation, software, formal analysis, writing-review and editing. J.R. Kowalczyk: Resources, data curation. T. Szczepański: Resources, data curation. U.P. Davé: Resources, formal analysis, writing-review and editing. A.E. Kulozik: Resources, data curation, software, formal analysis. S. Goossens: Conceptualization, data curation, supervision, writing-original draft, writing-review and editing. D.J. Curtis: Resources, data curation, formal analysis, supervision, writing-review and editing. T. Taghon: Conceptualization, resources, data curation, supervision, writing-review and editing. M. Dawidowska: Conceptualization, resources, data curation, software, supervision, writingreview and editing. S. Degerman: Conceptualization, resources, data curation, formal analysis, supervision, writing-review and editing. P. Van Vlierberghe: Conceptualization, resources, data curation, formal analysis, supervision, funding acquisition, validation, writingoriginal draft, project administration, writing-review and editing.

\section{Acknowledgments}

This work was supported by the following funding agencies: the European Research Council (StG-639784; to P. Van Vlierberghe), the Fund for Scientific Research Flanders, Kom op tegen Kanker (Stand up to Cancer; the Flemish Cancer Society), Stichting Tegen Kanker (STK), Kinderkankerfonds (a nonprofit childhood cancer foundation under Belgian law), Cancer Research Institute Ghent (CRIG), the National Science Center Poland (2017/24/T/NZ5/00359), the National Centre for Research and Development Poland (STRATEGMED3/304586/5/ NCBR/2017), the Swedish Childhood Foundation (PR2018-0064), the Medical Faculty of Umeå University, the Kempe Foundation, and the Lion's Cancer Research Foundation. R.S. Kotecha (NHMRC APP1142627) is supported by a fellowship from the National Health and Medical Research Council of Australia. M.R. Mansour is a Bloodwise Bennett Fellow. The Davé Lab was supported by R01CA207530 from the National Cancer Institute, I01BX001799 from the Department of Veterans Affairs, and the Indiana University School of Medicine Strategic Research Initiative. The computational resources and services used in this work were provided by the VSC (Flemish Supercomputer Center), funded by the Research Foundation - Flanders (FWO) and the Flemish Government-department EWI.

The costs of publication of this article were defrayed in part by the payment of page charges. This article must therefore be hereby marked advertisement in accordance with 18 U.S.C. Section 1734 solely to indicate this fact.

Received April 17, 2020; revised August 6, 2020; accepted September 15, 2020; published first September 23, 2020.

\section{REFERENCES}

1. Michalak EM, Burr ML, Bannister AJ, Dawson MA. The roles of DNA, RNA and histone methylation in ageing and cancer. Nat Rev Mol Cell Biol 2019;20:573-89.

2. Issa JP. CpG island methylator phenotype in cancer. Nat Rev Cancer 2004;4:988-93.

3. Borssen M, Palmqvist L, Karrman K, Abrahamsson J, Behrendtz M, Heldrup J, et al. Promoter DNA methylation pattern identifies prognostic subgroups in childhood T-cell acute lymphoblastic leukemia. PLoS One 2013;8:e65373. 
4. Kimura S, Seki M, Kawai T, Goto H, Yoshida K, Isobe T, et al. DNA methylation-based classification reveals difference between pediatric T-cell acute lymphoblastic leukemia and normal thymocytes. Leukemia 2019;34:1163-8.

5. Touzart A, Boissel N, Belhocine M, Smith C, Graux C, Latiri M, et al. Low level $\mathrm{CpG}$ island promoter methylation predicts a poor outcome in adult T-cell acute lymphoblastic leukemia. Haematologica 2019;105:1575-81.

6. Ferrando AA, Neuberg DS, Staunton J, Loh ML, Huard C, Raimondi SC, et al. Gene expression signatures define novel oncogenic pathways in $\mathrm{T}$ cell acute lymphoblastic leukemia. Cancer Cell 2002;1:75-87.

7. Homminga I, Pieters R, Langerak AW, de Rooi JJ, Stubbs A, Verstegen M, et al. Integrated transcript and genome analyses reveal NKX2-1 and MEF2C as potential oncogenes in T cell acute lymphoblastic leukemia. Cancer Cell 2011;19:484-97.

8. Szarzynska-Zawadzka B, Kunz JB, Sedek L, Kosmalska M, Zdon K, Biecek P, et al. PTEN abnormalities predict poor outcome in children with T-cell acute lymphoblastic leukemia treated according to ALL IC-BFM protocols. Am J Hematol 2019;94:E93-6.

9. Taghon T, Waegemans E, Van de Walle I. Notch signaling during human T cell development. Curr Top Microbiol Immunol 2012;360: 75-97.

10. Dolens AC, Durinck K, Lavaert M, Van der Meulen J, Velghe I, De Medts J, et al. Distinct Notch1 and BCL11B requirements mediate human gammadelta/alphabeta $\mathrm{T}$ cell development. EMBO Rep 2020;21:e49006

11. Haider Z, Larsson $P$, Landfors $M$, Kohn L, Schmiegelow K, Flaegstad T, et al. An integrated transcriptome analysis in T-cell acute lymphoblastic leukemia links DNA methylation subgroups to dysregulated TAL1 and ANTP homeobox gene expression. Cancer Med 2019;8:311-24

12. Zhang J, Ding L, Holmfeldt L, Wu G, Heatley SL, Payne-Turner D, et al. The genetic basis of early T-cell precursor acute lymphoblastic leukaemia. Nature 2012;481:157-63.

13. Liu Y, Easton J, Shao Y, Maciaszek J, Wang Z, Wilkinson MR, et al. The genomic landscape of pediatric and young adult T-lineage acute lymphoblastic leukemia. Nat Genet 2017;49:1211-8.

14. Kuleshov MV, Jones MR, Rouillard AD, Fernandez NF, Duan Q Wang Z, et al. Enrichr: a comprehensive gene set enrichment analysis web server 2016 update. Nucleic Acids Res 2016;44:W90-7.

15. Verboom K, Van Loocke W, Volders PJ, Decaesteker B, Cobos FA, Bornschein S, et al. A comprehensive inventory of TLX1 controlled long non-coding RNAs in T-cell acute lymphoblastic leukemia through polyA+ and total RNA sequencing. Haematologica 2018;103:e585-9.

16. Roels J, Kuchmiy A, De Decker M, Strubbe S, Lavaert M, Liang KL, et al. Distinct and temporary-restricted epigenetic mechanisms regulate human alphabeta and gammadelta $\mathrm{T}$ cell development. Nat Immunol 2020;21:1280-92.

17. Manser M, Sater MR, Schmid CD, Noreen F, Murbach M, Kuster N, et al. ELF-MF exposure affects the robustness of epigenetic programming during granulopoiesis. Sci Rep 2017;7:43345

18. Pope BD, Ryba T, Dileep V, Yue F, Wu W, Denas O, et al. Topologically associating domains are stable units of replication-timing regulation. Nature 2014;515:402-5

19. Huang J, Liu X, Li D, Shao Z, Cao H, Zhang Y, et al. Dynamic control of enhancer repertoires drives lineage and stage-specific transcription during hematopoiesis. Dev Cell 2016;36:9-23.

20. Minderjahn J, Schmidt A, Fuchs A, Schill R, Raithel J, Babina M, et al. Mechanisms governing the pioneering and redistribution capabilities of the non-classical pioneer PU.1. Nat Commun 2020;11:402.

21. Richter-Pechanska P, Kunz JB, Bornhauser B, von Knebel Doeberitz C, Rausch T, Erarslan-Uysal B, et al. PDX models recapitulate the genetic and epigenetic landscape of pediatric T-cell leukemia. EMBO Mol Med 2018;10:e9443.

22. Schmidl C, Rendeiro AF, Sheffield NC, Bock C. ChIPmentation: fast, robust, low-input ChIP-seq for histones and transcription factors. Nat Methods 2015;12:963-5

23. Beerman I, Bock C, Garrison BS, Smith ZD, Gu H, Meissner A, et al. Proliferation-dependent alterations of the DNA methylation landscape underlie hematopoietic stem cell aging. Cell Stem Cell 2013;12:413-25

24. Yang Z, Wong A, Kuh D, Paul DS, Rakyan VK, Leslie RD, et al. Correlation of an epigenetic mitotic clock with cancer risk. Genome Biol 2016;17:205.

25. Horvath S. DNA methylation age of human tissues and cell types. Genome Biol 2013;14:R115.

26. Smith S, Tripathi R, Goodings C, Cleveland S, Mathias E, HardawayJA, et al. LIM domain only-2 (LMO2) induces T-cell leukemia by two distinct pathways. PLoS One 2014;9:e85883.

27. Chen S, Wang Q, Yu H, Capitano ML, Vemula S, Nabinger SC, et al. Mutant p53 drives clonal hematopoiesis through modulating epigenetic pathway. Nat Commun 2019;10:5649.

28. Yashiro-Ohtani Y, Wang H, Zang C, Arnett KL, Bailis W, Ho Y, et al. Longrange enhancer activity determines Myc sensitivity to Notch inhibitors in T cell leukemia. Proc Natl Acad Sci U S A 2014;111:E4946-53.

29. Petkovich DA, Podolskiy DI, Lobanov AV, Lee SG, Miller RA, Gladyshev VN. Using DNA methylation profiling to evaluate biological age and longevity interventions. Cell Metab 2017;25:954-60.

30. Duchmann M, Itzykson R. Clinical update on hypomethylating agents. Int J Hematol 2019;110:161-99.

31. Benton CB, Thomas DA, Yang H, Ravandi F, Rytting M, O'Brien S, et al. Safety and clinical activity of 5-aza-2'-deoxycytidine (decitabine) with or without Hyper-CVAD in relapsed/refractory acute lymphocytic leukaemia. Br J Haematol 2014;167:356-65.

32. El Chaer F, Holtzman N, Binder E, Porter NC, Singh ZN, Koka M, et al. Durable remission with salvage decitabine and donor lymphocyte infusion (DLI) for relapsed early T-cell precursor ALL. Bone Marrow Transplant 2017;52:1583-4

33. Yang Y, Yao S, Zhang J, Yan Z, Chu J, Wang H, et al. Decitabinecontaining G-CSF priming regimen overcomes resistance of primary mediastinal neoplasm from early T-cell precursors to conventional chemotherapy: a case report. Onco Targets Ther 2019;12:7039-44.

34. Cui JK, Xiao Y, You Y, Shi W, Li Q, Luo Y, et al. Decitabine for relapsed acute lymphoblastic leukemia after allogeneic hematopoietic stem cell transplantation. J Huazhong Univ Sci Technolog Med Sci 2017;37:693-8.

35. Rahmat LT, Nguyen A, Abdulhaq H, Prakash S, Logan AC, Mannis GN. Venetoclax in combination with decitabine for relapsed T-cell acute lymphoblastic leukemia after allogeneic hematopoietic cell transplant. Case Rep Hematol 2018;2018:6092646.

36. Farhadfar N, Li Y, May WS, Adams CB. Venetoclax and decitabine for treatment of relapsed T-cell acute lymphoblastic leukemia: a case report and review of literature. Hematol Oncol Stem Cell Ther 2020;S1658-3876:30031-5.

37. Subramanian A, Tamayo P, Mootha VK, Mukherjee S, Ebert BL, Gillette MA, et al. Gene set enrichment analysis: a knowledge-based approach for interpreting genome-wide expression profiles. Proc Natl Acad Sci U S A 2005;102:15545-50.

38. De Decker M, Lavaert M, Roels J, Tilleman L, Vandekerckhove B, Leclercq G, et al. HES1 and HES4 have non-redundant roles downstream of Notch during early human T cell development. Haematologica 2020 Jan 9 [Epub ahead of print].

39. Hashimoto H, Wang D, Horton JR, Zhang X, Corces VG, Cheng X. Structural basis for the versatile and methylation-dependent binding of CTCF to DNA. Mol Cell 2017;66:711-20.

40. Teif VB, Beshnova DA, Vainshtein Y, Marth C, Mallm JP, Hofer T, et al. Nucleosome repositioning links DNA (de)methylation and differential CTCF binding during stem cell development. Genome Res 2014;24:1285-95

41. Hyle J, Zhang Y, Wright S, Xu B, Shao Y, Easton J, et al. Acute depletion of CTCF directly affects MYC regulation through loss of enhancer-promoter looping. Nucleic Acids Res 2019;47:6699-713.

42. Borssen M, Haider Z, Landfors M, Noren-Nystrom U, Schmiegelow K, Asberg AE, et al. DNA methylation adds prognostic value to minimal residual disease status in pediatric T-cell acute lymphoblastic leukemia. Pediatr Blood Cancer 2016;63:1185-92.

43. Wang C, Oshima M, Sato D, Matsui H, Kubota S, Aoyama K, et al. Ezh2 loss propagates hypermethylation at $\mathrm{T}$ cell differentiation-regulating 
genes to promote leukemic transformation. J Clin Invest 2018;128: 3872-86.

44. Danis E, Yamauchi T, Echanique K, Zhang X, Haladyna JN, Riedel SS, et al. Ezh2 controls an early hematopoietic program and growth and survival signaling in early $\mathrm{T}$ cell precursor acute lymphoblastic leukemia. Cell Rep 2016;14:1953-65.

45. Vire E, Brenner C, Deplus R, Blanchon L, Fraga M, Didelot C, et al. The Polycomb group protein EZH2 directly controls DNA methylation. Nature 2006;439:871-4.

46. Wu H, Zhang Y. Tet1 and 5-hydroxymethylation: a genome-wide view in mouse embryonic stem cells. Cell Cycle 2011;10:2428-36.

47. Zhou W, Yui MA, Williams BA, Yun J, Wold BJ, Cai L, et al. Singlecell analysis reveals regulatory gene expression dynamics leading to lineage commitment in early T cell development. Cell Syst 2019;9: 321-78.

48. Van Vlierberghe P, Ferrando A. The molecular basis of $\mathrm{T}$ cell acute lymphoblastic leukemia. J Clin Invest 2012;122:3398-406.

49. Cao L, Wang N, Pan J, Hu S, Zhao W, He H, et al. Clinical significance of microRNA-34b expression in pediatric acute leukemia. Mol Med Rep 2016;13:2777-84

50. Poole CJ, Zheng W, Lodh A, Yevtodiyenko A, Liefwalker D, Li H, et al. DNMT3B overexpression contributes to aberrant DNA methylation and MYC-driven tumor maintenance in T-ALL and Burkitt's lymphoma. Oncotarget 2017;8:76898-920.

51. Kowalczyk JR, Zawitkowska J, Lejman M, Drabko K, Samardakiewicz M, Matysiak M, et al. Long-term treatment results of Polish pediatric and adolescent patients enrolled in the ALL IC-BFM 2002 trial. Am J Hematol 2019;94:E307-10.

52. Krueger F, Andrews SR. Bismark: a flexible aligner and methylation caller for Bisulfite-Seq applications. Bioinformatics 2011;27:1571-2.

53. Assenov Y, Muller F, Lutsik P, Walter J, Lengauer T, Bock C. Comprehensive analysis of DNA methylation data with RnBeads. Nat Methods 2014;11:1138-40.

54. Zhu LJ. Integrative analysis of ChIP-chip and ChIP-seq dataset. Methods Mol Biol 2013;1067:105-24.
55. Dobin A, Davis CA, Schlesinger F, Drenkow J, Zaleski C, Jha S, et al. STAR: ultrafast universal RNA-seq aligner. Bioinformatics 2013;29: 15-21.

56. Zhou W, Laird PW, Shen H. Comprehensive characterization, annotation and innovative use of Infinium DNA methylation BeadChip probes. Nucleic Acids Res 2016;45:e22.

57. Gaunt TR, Shihab HA, Hemani G, Min JL, Woodward G, Lyttleton O, et al. Systematic identification of genetic influences on methylation across the human life course. Genome Biol 2016;17:61.

58. Zou LS, Erdos MR, Taylor DL, Chines PS, Varshney A, Parker SCJ, et al. BoostMe accurately predicts DNA methylation values in whole-genome bisulfite sequencing of multiple human tissues. BMC Genomics 2018;19:390.

59. Oki S, Ohta T, Shioi G, Hatanaka H, Ogasawara O, Okuda Y, et al. ChIP-Atlas: a data-mining suite powered by full integration of public ChIP-seq data. EMBO Rep 2018;19:e46255.

60. Ramírez F, Dündar F, Diehl S, Grüning BA, Manke T. deepTools: a flexible platform for exploring deep-sequencing data. Nucleic Acids Res 2014;42:W187-91.

61. Mootha VK, Lindgren CM, Eriksson KF, Subramanian A, Sihag S, Lehar J, et al. PGC-1alpha-responsive genes involved in oxidative phosphorylation are coordinately downregulated in human diabetes. Nat Genet 2003;34:267-73.

62. Huang da W, Sherman BT, Lempicki RA. Bioinformatics enrichment tools: paths toward the comprehensive functional analysis of large gene lists. Nucleic Acids Res 2009;37:1-13.

63. Huang DW, Sherman BT, Lempicki RA. Systematic and integrative analysis of large gene lists using DAVID bioinformatics resources. Nat Protoc 2009;4:44-57.

64. Chen EY, Tan CM, Kou Y, Duan Q, Wang Z, Meirelles GV, et al. Enrichr: interactive and collaborative HTML5 gene list enrichment analysis tool. BMC Bioinformatics 2013;14:128.

65. Love MI, Huber W, Anders S. Moderated estimation of fold change and dispersion for RNA-seq data with DESeq2. Genome Biol 2014; 15:550. 


\section{Aging of Preleukemic Thymocytes Drives CpG Island Hypermethylation in T-cell Acute Lymphoblastic Leukemia}

Juliette Roels, Morgan Thénoz, Bronislawa Szarzynska, et al.

Blood Cancer Discov 2020;1:274-289. Published OnlineFirst September 23, 2020.

Updated version Access the most recent version of this article at: doi: 10.1158/2643-3230.BCD-20-0059

Supplementary Access the most recent supplemental material at:

Material http://bloodcancerdiscov.aacrjournals.org/content/suppl/2020/09/23/2643-3230.BCD-20-0059 .DC1

Cited articles This article cites 64 articles, 6 of which you can access for free at:

http://bloodcancerdiscov.aacrjournals.org/content/1/3/274.full\#ref-list-1

E-mail alerts Sign up to receive free email-alerts related to this article or journal.

Reprints and To order reprints of this article or to subscribe to the journal, contact the AACR Publications

Subscriptions Department at pubs@aacr.org.

Permissions To request permission to re-use all or part of this article, use this link http://bloodcancerdiscov.aacrjournals.org/content/1/3/274.

Click on "Request Permissions" which will take you to the Copyright Clearance Center's (CCC)

Rightslink site. 